FHWA/IN/JTRP-2005/13

Final Report

STATEWIDE WIRELESS

COMMUNICATIONS PROJECT

Volume 1: Communications Field Tests for Satellite, Cellular, and Spread Spectrum Radio

James V. Krogmeier

Darcy M. Bullock

July 2008 


\section{TECHNICAL Summary}

INDOT Research

Technology Transfer and Project Implementation Information

TRB Subject Code: 54-8 Communications and Transportation Systems Management

Publication No.: FHWA/IN/JTRP-2005/13, SPR-2852

July 2008

Final Report

\section{Statewide Wireless Communications Project}

\section{Introduction}

The Indiana Department of Transportation (INDOT) is actively deploying digital radio technology across the state in the service of both temporary and permanent (fixed, portable, and mobile) data communications needs. There are also a number of major current and planned software applications that will significantly impact the need for data communications bandwidth at the far reaches of INDOT's network. The most pressing application is the SiteManager construction management system, which is being deployed now.

The problem addressed in this project was to carry out some of the research required for the development of wireless networking as outlined in INDOT's recently completed communications strategic plan. The work fell into four areas.

The first area (Task A) was to provide support for the test and evaluation of satellite networking in remote-site data communications involving the impending introduction of SiteManager software. The second area (Task B) was to evaluate ISM band spread spectrum radio for long range, multi-hop communications of telemetry and control for traffic signal controller management. The third area (Task C) was to research the problem of bicycle detection using inductive loop technology and the fourth and final area (Task D) was to do preliminary testing of a vehicle-infrastructure integration (VII) application in road condition monitoring. The work plan was developed and modified with the cooperation of the project study advisory committee as the project progressed.

Since the four tasks were essentially independent of each other, they are described separately below.

\section{Findings}

The major findings of this project are given below as organized by project task:

\section{Task A: Support for field tests of satellite communication service providers.}

Operated in client-server mode, with client running on a laptop in the field, SiteManager requires significant bi-directional wireless communications throughput for satisfactory operation (more than $300 \mathrm{kbps}$ ). This is very difficult to achieve over a wide area across the state. There are two factors that influence the achievable throughput. The first is the bandwidth of the link. The second is the roundtrip delay from client to server and the effect of delay upon the higher layer protocols of the application software. Satellite service providers have designed their systems to address the detrimental effect that delay has upon TCP/IP but they are unable to address protocol issues internal to particular application software.

Applications, which rely primarily upon TCP/IP, work well over satellite and are able to transform the large satellite bandwidth into application throughput even in the presence of delay. FTP is an example. However, other applications (such as SiteManager and Microsoft Outlook) are very "chatty" in that their design uses extensive sequential handshaking between client and server to execute certain tasks. These applications can't efficiently use a large bandwidth with large roundtrip delay. Their throughput would not be improved even if an 
entire satellite transponder were devoted to a single connection.

In the short term the only viable solution for SiteManager is to use terminal emulation (such as Citrix).

\section{Task B: Spread spectrum radio testing.}

In this task ISM band spread spectrum radio was evaluated to see if it could be used to build a statewide network to allow control and telemetry transmission from district and subdistrict offices to various traffic signal controller installations around the state. INDOT personnel had determined that traffic signal controller installations are deployed sufficiently densely around the state that if spread spectrum products could achieve around 10 mile transmission ranges, then connections could be made in a multi-hop fashion from one site to the next ultimately providing much longer range communications.

After testing and evaluation it was determined that this idea is not yet feasible. There are two problems that would need to be overcome. First, given Indiana's rolling topography and significant vegetation it is not possible to obtain line of sight transmission with antenna heights that correspond to antenna mounting on signal arms. In our range tests, 3 miles was the effective range limit obtained. Second, the network design would be relatively complicated and the network configuration flexibility offered by current products is insufficient.

\section{Task C: Research on bicycle detection with inductive loops.}

In this task we have developed a capability to numerically compute the detection zone for bicycles as a function of the loop geometry, loop winding, and loop detector sensitivity setting. The numerical method has been calibrated and verified by field measurement.

Our findings were:

a. To within reasonable engineering accuracy, circular and octagonal loops offer the same detection zones for bicycles.

b. Both circular and octagonal loops have significant deadzones such that a bicycle traveling through the center of a loop would not be detected at the standard sensitivity setting.

c. Bicycle detection is seriously degraded by the practice of wiring four loops in series and/or by paving over the loops (and thereby increasing loop depth under the pavement).

\section{Task D: Prototype of a vehicle- infrastructure integration (VII) system.}

In this task a van was instrumented with acceleration sensors, GPS, data acquisition, data logging, and wireless communications to enable preliminary testing of a VII application for road condition monitoring. A rudimentary capability to produce maps indicating the locations of significant ride disturbances was demonstrated.

\section{Implementation}

The main recommendations for implementation are given below organized by project task.

\section{Task A: Support for field tests of satellite communication service providers.}

SiteManager should be implemented via terminal emulation with the client software running on computers connected via a high bandwidth, low delay network. This recommendation is being implemented by INDOT. In addition:
a. INDOT should make recommendations to AASHTO that the communications protocol design of SiteManager and

other application software be improved to account for delay in satellite networks.

b. Further research should be carried out in support of the terminal emulation solution for SiteManager because using terminal emulation will require provision for communications anywhere in the worksite where the field engineer wants to use the program.

\section{Task B: Spread spectrum radio testing.}


Equipment providers are continually introducing improvements in spread spectrum radio technology. In particular, IP radio technology is available now and will likely grow in importance to INDOT. Therefore, the spread spectrum radio testbed should be maintained to serve in evaluation of these radios as the need arises.

3. Task C: Research on bicycle detection with inductive loops.

The recommendations for implementation are:

a. Pavement markings should be placed on the road surface at selected intersections to indicate the location of best bicycle detection.

b. In situations where bicycle detection is important, INDOT should consider wiring the loop closest to the stop bar on a separate loop detector rather than wiring it in series with the other three loops.

c. When using pave-over loops detection sensitivity should be increased over the standard default setting because deeper loops compound the difficulty of bicycle detection.

\section{Task D: Prototype of a vehicle- infrastructure integration (VII) system.}

Further development should be undertaken of the VII testbed vehicle. Additional items to consider are the introduction of additional sensors (suspension travel, steering, temperature, and windshield wiper status) and the optimal filtering of sensor data for detection of ride anomalies.

\section{Contacts}

For more information:

\section{Prof. James V. Krogmeier}

Co-Principal Investigator

School of Electrical and Computer Engineering

Purdue University

West Lafayette IN 47907

Phone: (765) 494-3530

Fax: (765) 494-3358

\section{Prof. Darcy Bullock}

Co-Principal Investigator

School of Civil Engineering

Purdue University

West Lafayette, IN 47907

Phone: (765) 494-2226

Fax: (765) 496-7996

\section{Indiana Department of Transportation}

Division of Research

1205 Montgomery Street

P.O. Box 2279

West Lafayette, IN 47906

Phone: (765) 463-1521

Fax: (765) 497-1665

\section{Purdue University}

Joint Transportation Research Program

School of Civil Engineering

West Lafayette, IN 47907-1284

Phone: (765) 494-9310

Fax: (765) 496-7996

http:d/www.purdue.edu/jtrp 
Final Report

FHWA/IN/JTRP-2005/13

\title{
STATEWIDE WIRELESS COMMUNICATIONS PROJECT \\ Volume 1: Communication Field Tests for Satellite, Cellular, and Spread Spectrum Radio
}

\author{
By \\ James V. Krogmeier \\ Associate Professor \\ School of Electrical and Computer Engineering \\ Purdue University \\ and \\ Darcy M. Bullock \\ Professor \\ School of Civil Engineering \\ Purdue University \\ Joint Transportation Research Program \\ Project No. C-36-75O \\ File No. 8-9-15 \\ SPR-2852

\begin{abstract}
Conducted in Cooperation with the Indiana Department of Transportation

Federal Highway Administration
\end{abstract} \\ and the U.S. Department of Transportation
}

The contents of this report reflect the views of the authors, who are responsible for the facts and the accuracy of the data presented herein. The contents do not necessarily reflect the official views or policies of the Indiana Department of Transportation or the Federal Highway Administration at the time of publication. This report does not constitute a standard, specification, or regulation.

Purdue University

West Lafayette, IN 47907

July 2008 
TECHNICAL REPORT STANDARD TITLE PAGE

\begin{tabular}{|c|c|c|}
\hline $\begin{array}{l}\text { 1. Report No. } \\
\text { FHWA/IN/JTRP-2005/13 }\end{array}$ & 2. Government Accession No. & 3. Recipient's Catalog No. \\
\hline \multirow{2}{*}{\multicolumn{2}{|c|}{$\begin{array}{l}\text { 4. Title and Subtitle } \\
\text { Statewide Wireless Communications Project }\end{array}$}} & $\begin{array}{l}\text { 5. } \text { Report Date } \\
\text { July } 2008\end{array}$ \\
\hline & & 6. Performing Organization Code \\
\hline \multicolumn{2}{|l|}{$\begin{array}{l}\text { 7. Author(s) } \\
\text { J. V. Krogmeier and D. M. Bullock }\end{array}$} & $\begin{array}{l}\text { 8. Performing Organization Report No. } \\
\text { FHWA/IN/JTRP-2005/13 }\end{array}$ \\
\hline \multirow{2}{*}{\multicolumn{2}{|c|}{$\begin{array}{l}\text { 9. Performing Organization Name and Address } \\
\text { Joint Transportation Research Program } \\
1284 \text { Civil Engineering Building } \\
\text { Purdue University } \\
\text { West Lafayette, IN 47907-1284 }\end{array}$}} & 10. Work Unit No. \\
\hline & & $\begin{array}{l}\text { 11. Contract or Grant No. } \\
\text { SPR-2852 }\end{array}$ \\
\hline \multirow{2}{*}{\multicolumn{2}{|c|}{$\begin{array}{l}\text { 12. Sponsoring Agency Name and Address } \\
\text { Indiana Department of Transportation } \\
\text { State Office Building } \\
100 \text { North Senate Avenue } \\
\text { Indianapolis, IN } 46204\end{array}$}} & $\begin{array}{l}\text { 13. Type of Report and Period Covered } \\
\text { Final Report }\end{array}$ \\
\hline & & 14. Sponsoring Agency Code \\
\hline
\end{tabular}

15. Supplementary Notes

Prepared in cooperation with the Indiana Department of Transportation and Federal Highway Administration.

\section{Abstract}

The Statewide Wireless Communications Project was an umbrella project intended to support various INDOT activities in the area of wireless communications. As these activities were conducted independently the report for the project is organized into three volumes. Volume 1 contains the results of satellite and cellular communications field testing undertaken in support of INDOT's SiteManager application. Volume 1 also contains the results of an evaluation of spread spectrum radios for long-range communications. Volume 2 contains the results of detection zone evaluation for loop detection of bicycles and the results of testing algorithms for travel time estimation using vehicle re-identification based on inductive and micro-loop signatures. Finally, Volume 3 contains the results of preliminary testing of a vehicle-infrastructure integration application in road condition monitoring. In Volume 1 we found that SiteManager could not be adequately run over a satellite link because the long round trip delay of the communication link negatively interacted with SiteManager's internal client-server communications protocol to severely reduce overall throughput. A solution to the problem was to use terminal emulation in the field with the client software running on a computer connected to the server via a high bandwidth, low delay link. The downside to the terminal emulation approach is that it requires that the field engineer have a communication link wherever the application is run. In Volume 1 we also found that current generation spread spectrum radio ranges in Indiana topography with antenna heights corresponding to signal arm mounting were on the order of 3 miles. This was too short by a factor of 3 to support a multihop network for traffic signal control and telemetry. In Volume 2 we developed a numerical technique for mapping the bicycle detection zones of loop detectors. A number of recommendations were made concerning loop geometry, depth, detector sensitivity, and pavement markings for purposes of improving bicycle detection. We also developed algorithms for travel time estimation based on vehicle signatures captured from commercially available inductive and micro-loop detector cards. The travel time estimation algorithms were field tested and show promise. In Volume 3 a prototype road condition monitoring system was built upon a passenger van platform and preliminary field testing and data analysis was done. Algorithms were developed to address positional uncertainties present in GPS measurements in order to allow the averaging of data taken in multiple independent runs. The results were

also field tested using INDOT’s Laser Profiling vehicle.

\begin{tabular}{|c|c|c|c|c|}
\hline \multicolumn{2}{|c|}{$\begin{array}{l}\text { 17. Key Words } \\
\text { satellite communications, TCP/IP, spread spectrum radios, } \\
\text { inductive and micro-loop detection, vehicle-infrastructure } \\
\text { integration. }\end{array}$} & \multicolumn{3}{|c|}{$\begin{array}{l}\text { 18. Distribution Statement } \\
\text { No restrictions. This document is available to the public through the } \\
\text { National Technical Information Service, Springfield, VA } 22161\end{array}$} \\
\hline $\begin{array}{c}\text { 19. Security Classif. (of this report) } \\
\text { Unclassified }\end{array}$ & \multicolumn{2}{|c|}{$\begin{array}{l}\text { 20. Security Classif. (of this page) } \\
\text { Unclassified }\end{array}$} & $\begin{array}{l}\text { 21. No. of Pages } \\
\text { Vol } 1-42 \\
\text { Vol } 2-37 \\
\text { Vol } 3-31\end{array}$ & 22. Price \\
\hline
\end{tabular}




\begin{abstract}
:
The Statewide Wireless Communications Project was an umbrella project intended to support various INDOT activities in the area of wireless communications. As these activities were conducted independently the report for the project is organized into three volumes. Volume 1 contains the results of satellite and cellular communications field testing undertaken in support of INDOT's SiteManager application. Volume 1 also contains the results of an evaluation of spread spectrum radios for long-range communications. Volume 2 contains the results of detection zone evaluation for loop detection of bicycles and the results of testing algorithms for travel time estimation using vehicle re-identification based on inductive and micro-loop signatures. Finally, Volume 3 contains the results of preliminary testing of a vehicle-infrastructure integration application in road condition monitoring.
\end{abstract}

In Volume 1 we found that SiteManager could not be adequately run over a satellite link because the long round trip delay of the communication link negatively interacted with SiteManager's internal client-server communications protocol to severely reduce overall throughput. A solution to the problem was to use terminal emulation in the field with the client software running on a computer connected to the server via a high bandwidth, low delay link. The downside to the terminal emulation approach is that it requires that the field engineer have a communication link wherever the application is run. In Volume 1 we also found that current generation spread spectrum radio ranges in Indiana topography with antenna heights corresponding to signal arm mounting were on the order of 3 miles. This was too short by a factor of 3 to support a multihop network for traffic signal control and telemetry.

In Volume 2 we developed a numerical technique for mapping the bicycle detection zones of loop detectors. A number of recommendations were made concerning loop geometry, depth, detector sensitivity, and pavement markings for purposes of improving bicycle detection. We also developed algorithms for travel time estimation based on vehicle signatures captured from commercially available inductive and micro-loop detector cards. The travel time estimation algorithms were field tested and show promise.

In Volume 3 a prototype road condition monitoring system was built upon a passenger van platform and preliminary field testing and data analysis was done. Algorithms were developed to address positional uncertainties present in GPS measurements in order to allow the averaging of data taken in multiple independent runs. The results were also field tested using INDOT's Laser Profiling vehicle.

Keywords: satellite communications, TCP/IP, spread spectrum radios, inductive and micro-loop detection, vehicle-infrastructure integration. 


\section{TABLE OF CONTENTS}

Section

Page

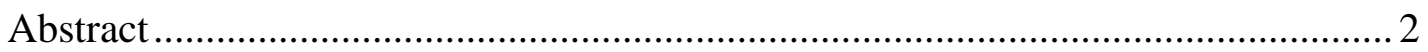

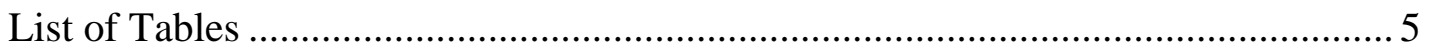

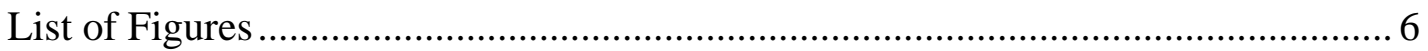

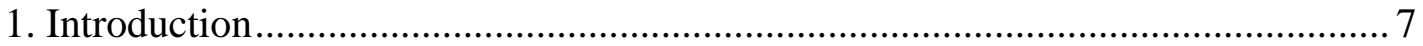

1.1 Summary of Communications Needs from Consultant's Study .................... 7

1.2 Some Background on Wireless Networking ................................................ 7

1.2.1 Cellular Telephony.................................................................... 8

1.2.2 Satellite Data Services ...................................................................... 9

1.2.3 Microwave Radio Systems .............................................................. 10

1.2.4 Spread Spectrum Radio in Unlicensed Bands ................................... 10

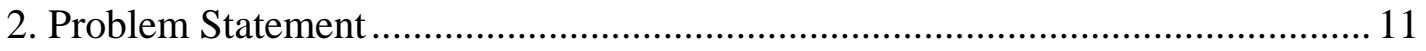

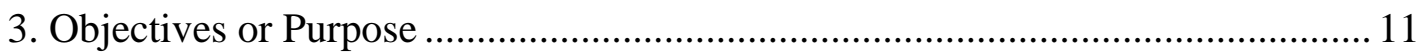

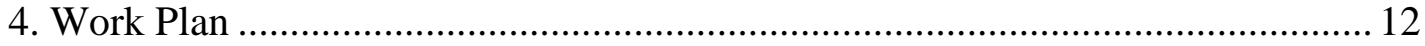

4.1 Task A: Support for Field Tests of Satellite Communication

Service Providers ................................................................................. 12

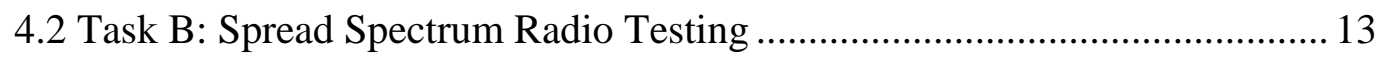

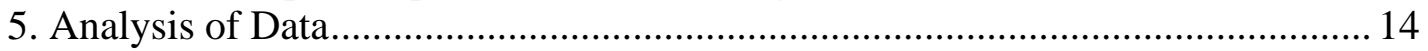

5.1 Task A: Support for Field Tests of Satellite Communication

Service Providers ................................................................................ 14

5.1.1 Satellite Communications Background............................................ 14

5.1.1.1 TCP Congestion Control....................................................... 14

5.1.1.2 Alternative Approaches ....................................................... 16

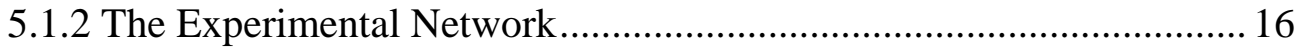

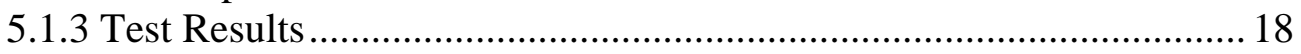

5.1.3.1 T1 Terrestrial versus 384 kbps / 384 kbps Satellite................... 19

5.1.3.2 Non-ALOHA versus ALOHA ................................................... 22

5.1.3.3 384 kbps / 384 kbps versus 1.544 Mbps / 512 kbps ................... 22

5.1.3.4 T1 versus 384 kbps / 384 kbps ALOHA versus Dialup.............. 23

5.1.3.5 Service Provider A versus B .................................................... 27

5.1.4 Remote Terminal Emulation......................................................... 27

5.2 Task B: Spread Spectrum Radio Testing ..................................................... 30

5.2.1 Summary of Specifications of Tested Radio ..................................... 30

5.2.2 Theoretical Range Calculation from Specifications and Propagation

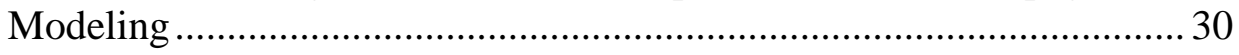

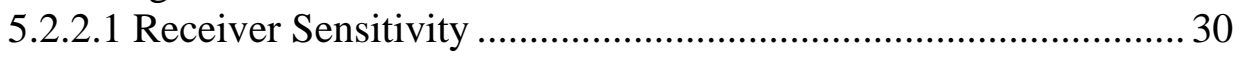

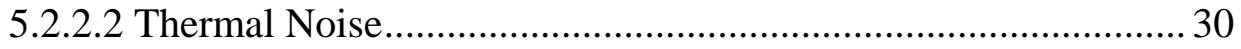

5.2.2.3 SNR to Achieve a Target BER ................................................ 31

5.2.2.4 Noise Figure....................................................................... 31

5.2.2.5 Link Budget Analysis for Free Space Propagation..................... 31

5.2.2.6 Link Budget Analysis for a Two-Ray Model and Fading........... 32 


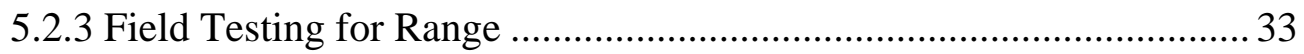

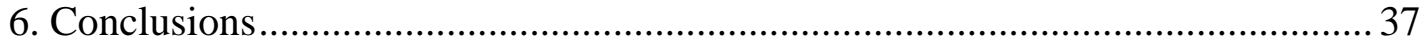

6.1 Task A: Support for Field Tests of Satellite Communication

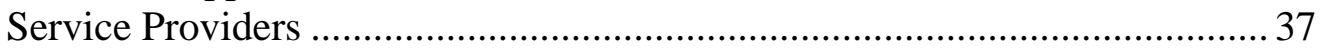

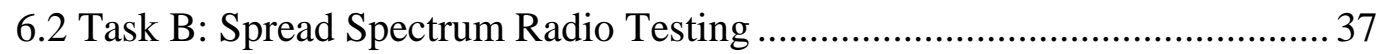

7. Recommendations and Implementation Suggestions ........................................ 38

7.1 Task A: Support for Field Tests of Satellite Communication

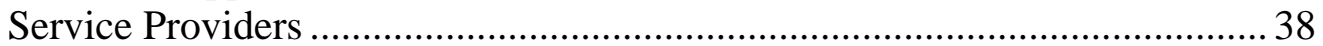

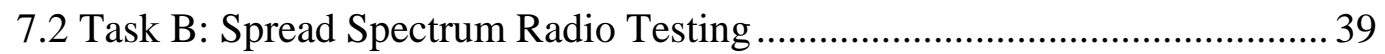

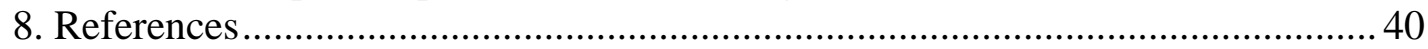




\section{LIST OF TABLES}

Table

Page

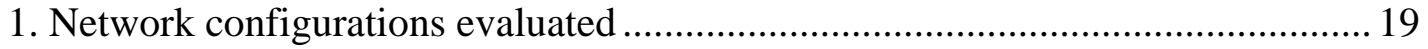

2. Description of exercises conducted with Outlook, Explorer, FTP, and

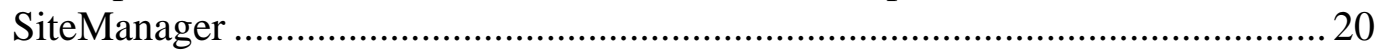

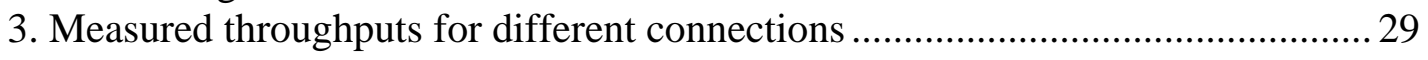

4. Observed signal/noise strength and throughput ............................................ 34 


\section{LIST OF FIGURES}

Figure Page

1. Verizon 1x RTT national access coverage map for Indiana ................................ 9

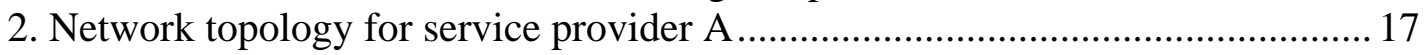

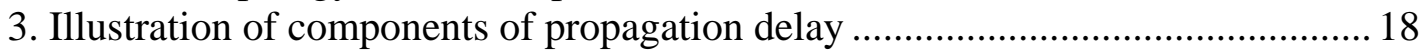

4. T1 terrestrial versus $384 \mathrm{kbps} / 384 \mathrm{kbps}$ satellite ............................................ 21

5. Ratio of $384 \mathrm{kbps} / 384 \mathrm{kbps}$ response time to $\mathrm{T} 1$ response time......................... 22

6. Ratios of $384 \mathrm{kbps} / 384 \mathrm{kbps}$ (first column) and 384 kbps / 384 kbps with

ALOHA (second column) to T1 response time ................................................ 23

7. $384 \mathrm{kbps} / 384 \mathrm{kbps}$ versus 1.544 Mbps / 512 kbps ............................................ 24

8. Performances of T1 versus $384 \mathrm{kbps} / 384 \mathrm{kbps}$ ALOHA versus dialup .............. 25

9. Throughput observed for $384 \mathrm{kbps} / 384$ kbps with ALOHA ............................... 25

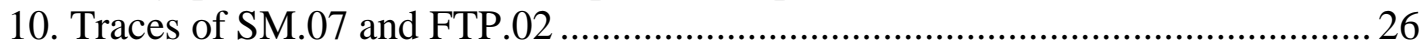

11. Predicted response time for SM.07 as bandwidth (QoS) provided varies .......... 27

12. Service provider A versus service provider B ............................................ 28

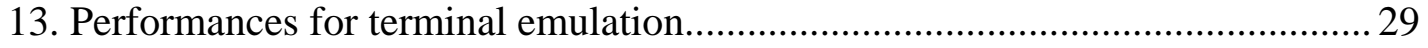

14. Performance for non-Citrix applications using different connections ................ 29

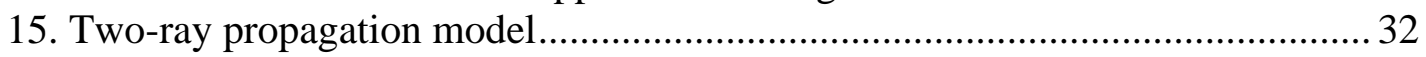

16. Remote radio equipment in van and antenna as hoisted by INDOT boom

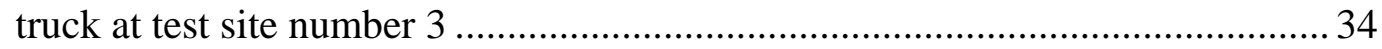

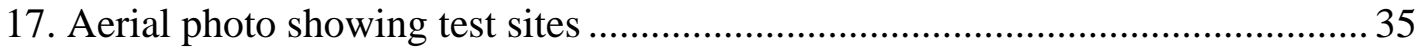

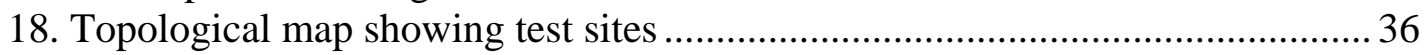




\section{Introduction}

The Indiana Department of Transportation (INDOT) is actively deploying digital radio technology across the state in the service of both temporary and permanent (fixed, portable, and mobile) data communications needs. Additional upgrades of wired (both fiber and copper) networking are also underway. Some of the INDOT projects at the forefront of wired and wireless network expansion include advanced traffic management systems (ATMS) in Indianapolis and on the Borman Expressway, the Virtual Weigh Station Project, and coordinated traffic signal systems in several cities. INDOT also participates in Project Hoosier SAFE-T, an initiative of Indiana's local, state, and federal public safety agencies, to implement a new $800 \mathrm{MHz}$ wireless system for radio communications (voice and data).

There are also a number of major current and planned software applications that will significantly impact the need for data communications bandwidth at the far reaches of INDOT's network. The most pressing application is the SiteManager construction management system, which is being deployed now. SiteManager will be located in semipermanent project trailers where "hard-wire" connections with land lines may not be practical, yet it also requires a two-way communications link of about $384 \mathrm{kbps}$ for best operation.

Faced with a challenging collection of current and future networking needs INDOT retained the consulting firm Edwards \& Kelcey, Inc., to produce a communications strategic plan. The plan was completed and approved in October 2002. A significant part of the work completed in this project has grown out of the recommendations made in the Edwards \& Kelcey report [EK 2002].

\subsection{Summary of Communications Needs From Consultant's Study}

One of the major observations of the study [EK 2002] was that network bandwidth available in the far reaches of the INDOT network is far from adequate to enable the rollout of new application software with its reliance upon client-server networked communications. Of particular concern was the plan to deploy SiteManager, which needs a bi-directional link between client and server of approximately 384 kbps for best operation. As planned at the time, SiteManager would be run on a client located on the construction site and so out of reach of INDOT's wired network (at least in many cases this would be so). There are also a number of other client-server applications that require significant network bandwidth at least out to the districts and sub-districts.

\subsection{Some Background on Wireless Networking}

Edwards and Kelcey [EK 2002] looked into a number of technologies for application in INDOT's communications network. The most promising were cellular telephony, satellite data services, and unlicensed spread spectrum radio for both distribution and 
network backbone. In this section, we review briefly these technologies and comment on the recommendations of the strategic plan.

\subsubsection{Cellular Telephony}

Cellular telephone systems in the United States are practically all digital at the present time. While the architecture of current (so-called second-generation) systems is optimized for voice, all major service providers have been actively rolling out data services, which support text messaging, email, and some low-quality still image and video transmission. The coverage for these advanced services is far from complete although progress is steady. There are two competing technologies available in the United States (both are available in Indiana as well). The market share leader is based upon the pan-European standard GSM and the data service associated with it is based upon the GPRS standard. GSM/GPRS uses a TDMA/FDMA multiple access scheme with very power efficient modulation at the physical layer. The power efficiency, leading to very good talk-times, is a major advantage. GSM/GPRS systems can provide a single user data rates up to $115 \mathrm{kbps}$ [Liu 2002]. But network planning for these systems is such that wide area coverage is first provided for only basic services (e.g., voice and low rate data for text messaging) with high rate data services being provided in areas where high population density can justify the economics for the service provider. The second major technology is based upon CDMA (actually, on Qualcomm's version of CDMA). The high rate data service associated is called 3G-1X EVDO (evolution data only) and is rapidly being rolled out across the country including in Indiana [Bi 2002]. It is an economical evolutionary path for IS-95 CDMA networks to offer high rate data services with IPbased packet switching. Data rates available on the traffic channel range from $38.4 \mathrm{kbps}$ to $2457.6 \mathrm{kbps}$, although the actual throughput per user is a decreasing function of the number of active users in a sector and is also highly dependent upon channel properties such as slow and fast envelope fading. A more realistic throughput per user is in the range of a few tens of kbps.

In Indiana Verizon Wireless offers their so-called express network service based upon the 3G-1X EVDO technology. They claim average speeds in the range $40-60 \mathrm{kbps}$ with bursts as high as $144 \mathrm{kbps}$. Verizon offers calling plans specifically tailored to data users as pricing is dependent upon actual data volume (rather than talk time). T-Mobile and AT\&T Wireless offer data services in Indiana derived from GSM/GPRS technology. These networks will typically have data rates lower than the CDMA technologies. In all cases, the guaranteed data rates are not sufficient to support INDOT's SiteManager application. Figure 1 shows the current advertised coverage map for Verizon's high speed cellular data service in Indiana. Although coverage appears good in the map on the course scale shown, it is still quite likely that data service is inadequate for demanding applications in a large number of remote locations. 


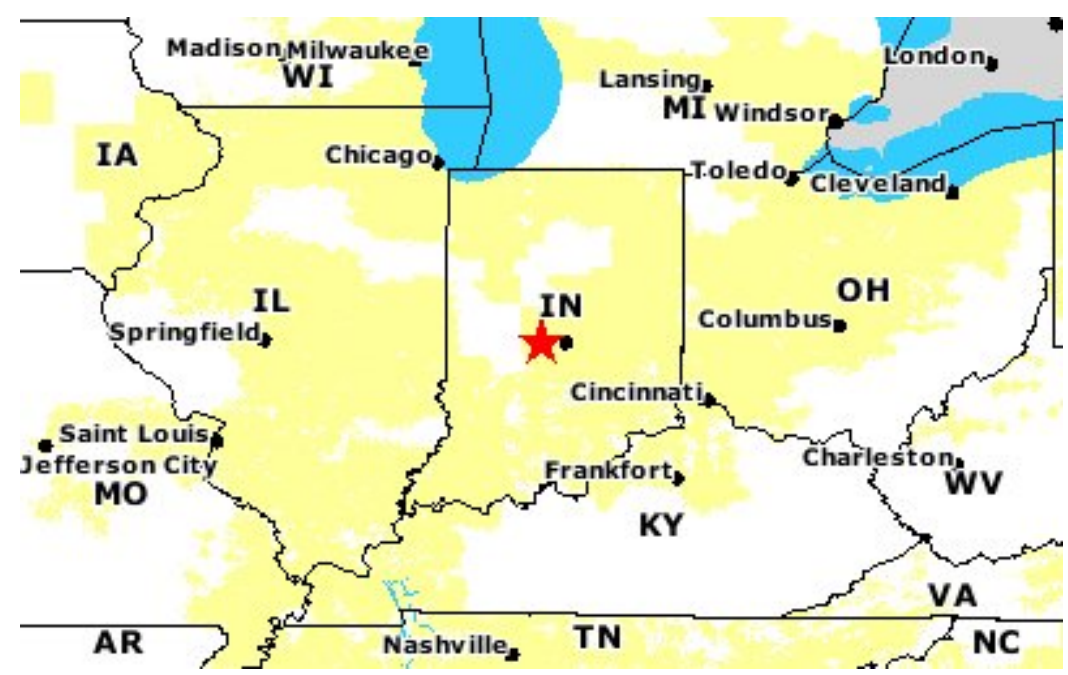

Figure 1: Verizon 1x RTT national access coverage map for Indiana.

\subsubsection{Satellite Data Services}

There are currently a number of satellite data services, which offer wide area coverage encompassing the entire state. These services use satellite transponders in either C-band $(6 / 4 \mathrm{GHz})$ or Ku-band $(14 / 11 \mathrm{GHz})$ and offer economical VSAT (very small aperture terminals) to government, corporate, and private users. New services will undoubtedly be offered in the Ka-band (30/20 GHz) over the next 5 - 10 years. As C- and Ku-band frequencies and geo-synchronous (GEO) orbits over North America become more crowded, the trend is naturally toward higher carrier frequencies. At higher carriers it becomes possible to design high gain fixed antennas of much smaller physical size, which is a definite advantage from a cost viewpoint. Similarly, more bandwidth is available for data communications in the Ka-band. On the downside are more difficult problems in the technology of high power amplifiers and filters in Ka-band (which will ultimately be alleviated) and the problem of higher attenuation in rainfall (which has the effect of lowering signal-to-noise ratio and hence lowering fade margins and ultimately the link availability). Rain attenuation in decibels (dB) increases roughly as the square of frequency (above $10 \mathrm{GHz}$ ), in fact, rain attenuation is about four times larger in the Kaband than in the Ku-band [Pratt 2003]. This physical problem will not be easily alleviated with improvements in microwave technology and it is therefore likely that the availability or reliability of Ka-band systems will never match that of C-band systems. C-band links can be designed to achieve 99.99 percent availability, which corresponds to an average outage time of 40 minutes per year. Ka-band links will achieve about 99.5 percent availability for an average outage time of 40 hours per year. Still this link quality may be acceptable in many INDOT applications.

The other major consideration in satellite data communications has to do with networking and how the satellite link can be incorporated in a seamless and user-transparent fashion. The most common configuration for the satellite portion of a network is a star, where all data traffic from remote VSAT terminals must take the hop up to a satellite and back 
down to a hub station, which is typically connected to the Internet or a private backbone. The one-way delay in a typical GEO link is 260 ms compared to a one-way delay of only $13 \mathrm{~ms}$ for a 4000-km terrestrial link. This added delay can cause standard protocols such as TCP/IP to be very inefficient (because timeout windows are not designed for long delays and the transmission rate setting procedures can be very inefficient with long delay). Most satellite providers address these networking issues by doing protocol conversions for the satellite portion of the link and "spoofing" in order to retain the transparent nature of the connection.

The Edwards and Kelcey study [EK 2003] identified three satellite service providers for INDOT's remote location and application requirements. It was recommended that Tachyon, iDirect, and ViaSat be examined for demonstration testing.

\subsubsection{Microwave Radio Systems}

These refer to systems mostly intended to replace point-to-point wired communication links operating at very high speeds (i.e., equivalent to multiple T1 rates). These systems can be economical compared to leased lines [Cohen 2003]. Advantages include their lowered installation costs compared to cable laying costs and their enhanced reliability (i.e., there is no chance of cable cutting). Systems rely upon highly directional antennas, high-order modulations (e.g., 64-QAM and higher), and polarization diversity in order to deliver very high bandwidth efficiency. Systems like this would normally be very sensitive to interference but careful antenna engineering and frequency planning will mitigate problems. Note that classical microwave systems use dedicated and licensed frequencies. A number of manufacturers of these technologies exist including Harris Microwave and Alcatel.

\subsubsection{Spread Spectrum Radio in Unlicensed Bands}

There are a very large number of manufacturers of spread spectrum radio systems intended for traffic systems applications. Broadcast is typically done in unlicensed bands around 900, 2400, and $5800 \mathrm{MHz}$. INDOT has a fair experience with these systems as they've been in use for some time in coordinated traffic signal control systems and other projects. In addition, a spread spectrum network testbed exists at Purdue, which is operated in cooperation with the Harold L. Michael Traffic Operations Laboratory. Typical data rates on these systems are on the order of a few tens of kbps.

Spread spectrum radio is also often used for high-bandwidth point-to-point radio links. INDOT has long been using the technology in this context on the Borman Expressway ATMS [Krogmeier 1996]. Typical data rates for these systems are from a few hundred Kbps to T1 rates. INDOT has experience with radios manufactured by Cylink, Western Multiplex, and others. This technology could be used to extend the microwave capacity of the ISP/INDOT backbone to the districts, sub-districts, and other convenient locations. 


\section{Problem Statement}

INDOT's communications strategic plan [EK 2002] contained a number of recommendations in the area of wireless networking. The strategic plan envisioned a phased migration from current leased lines to a broadband communications network incorporating a mix of wired and wireless technologies. Certain recommendations were made, those most relevant to this project are repeated below.

1. It was recommended that INDOT pursue the upgrade and extension of the state police digital microwave system to provide additional backbone connectivity for INDOT's network. The state police microwave backbone currently has a capacity of two DS3s and can be expanded by an additional DS3 (44.736 Mbps), which could be used by INDOT.

2. It was recommended that INDOT consider commercial wireless data and satellite services for mobile/remote access to service a wide range of communications needs including SiteManager and telemetry and control for weigh-in-motion station locations and intelligent signs.

3. It was recommended that INDOT develop shared resources for radio tower space and reserve space on state-owned towers for installation of digital microwave and spread spectrum radio antennas. Furthermore, it was advised to reserve bandwidth on TMC, ISP, and SAFE-T networks for INDOT use.

The problem addressed in the first volume of this project report was to carry out some of the research required for the development of wireless networking as outlined in the communications strategic plan. The work fell into two topic areas, each divided further into tasks. The first topic was to provide support for the test and evaluation of satellite networking in remote-site data communications involving the impending introduction of SiteManage software. The second topic was to evaluate ISM band spread spectrum radio for long range, multi-hop communications of telemetry and control for traffic signal controller management. The work plan was developed and modified with the cooperation of the project study advisory committee as the project progressed.

\section{Objectives or Purpose}

INDOT's communications strategic plan [EK 2002] suggests the building of a multifaceted network involving wired (fiber and copper) and wireless (satellite, microwave, spread spectrum, and cellular) components, where a particular technology is chosen based upon availability in a particular location and the associated costs of use. A high level analysis of the options was carried out along with some cost/benefit discussion. But for the most part, the study left a hard numbers analysis including field testing for follow on work. The objective of this project was to provide some of the hard data required to make engineering choices as INDOT's network is expanded. The research involved field testing of satellite data communications services to identify which services 
could support the needs of the SiteManager application. Field testing of spread spectrum and unlicensed microwave radio systems was also be carried out as part of continued operation of the testbed already established at Purdue.

\section{Work Plan}

The work of Volume 1 of the project was carried out in two major tasks described below. Since the tasks were essentially independent of each other, they will be described separately in this section and the next.

\subsection{Task A: Support for Field Tests of Satellite Communication Service Providers}

A follow up report to the communications strategic plan [EK 2003] identified three providers of satellite data services for possible field tests. These were Tachyon, iDirect, and ViaSat. In addition, the Indiana Division of Natural Resources (DNR) has DirectPC and SpaceNet satellite systems in place at its Fort Harrison State Park location. The principal investigators of this project and INDOT personnel were able to observe these systems and run tests on the uplink and downlink capacity. In two tests DirectPC achieved uplink/downlink speeds of 37.4/124.7 kbps (using Speed-Test.Net) and 9.8/171.3 kbps (using BandWidthPlace). The SpaceNet system achieved an uplink speed of $52.8 \mathrm{kbps}$ (using BandWidthPlace). These two data rate testing methods are actually web pages that send and receive test files between the web page server and the user's location. Part of the link was made up of the DirectPC and SpaceNet satellite links, respectively, but the nature of the remaining links was unknown and would often change from connection to connection due to routing chosen over the Internet. In addition, test results will also depend on congestion over the internet connection. Nevertheless, the relatively poor performance of DirectPC is almost certainly due to an oversubscribed uplink.

The performance/cost quotations from Tachyon, iDirect, and ViaSat (as given in [EK 2003]) are shown in the table below. Installation costs are not shown but run between \$500 and \$1000 per site. Also, the iDirect system requires the installation of a hub in Indianapolis at a cost of $\$ 274,000$.

\begin{tabular}{|c|c|c|c|c|}
\hline Provider & Uplink (kbps) & $\begin{array}{l}\text { Downlink } \\
\text { (kbps) }\end{array}$ & Monthly Lease & $\begin{array}{l}\text { Equipment } \\
\text { Costs }\end{array}$ \\
\hline Tachyon & 256 & 1544 & $\$ 2100$ & \$4999 per site \\
\hline iDirect & $128-256$ & 1500 & $\begin{array}{l}\$ 267 \text { - } \$ 400 \\
\text { (for } 300 \text { units) }\end{array}$ & $\begin{array}{l}\$ 2795 \text { - } \$ 3800 \\
\text { per site }\end{array}$ \\
\hline ViaSat & 128 & 384 & $\$ 139$ - \$159 & \$1995 per site \\
\hline
\end{tabular}

The most economical (in terms of monthly lease and customer equipment costs) VSAT systems are based upon digital video broadcast (DVB) technology. Since DVB service was not designed with data communications in mind it is not well-optimized for it. In DVB-based services data is encapsulated in an MPEG frame (even on the return channel) 
and this can lead to some inefficiencies in channel utilization. For example, when IP packets are transmitted over the DVB system, they must often be broken up to fit into MPEG cells of size 188 bytes. Usually, the DVB-based systems have a much higher down capacity than that for upload. In addition, the upload link is usually TDMA but based on Slotted Aloha and it therefore suffers dramatically under heavy utilization. The DirectPC and ViaSat LinkStar systems are based upon DVB. Their main advantage is the low cost of customer equipment and monthly leases. From the DNR testing and ViaSat's quotation it did not appear that these DVB-based solutions would suffice for INDOT's SiteManager application.

There was a need to gain experience with these systems and so a series of operational tests was planned. The results of these tests are described in the next section.

\subsection{Task B: Spread Spectrum Radio Testing}

This task was undertaken at the request of the project study advisory committee after the project proposal was written. In discussions with a traffic signal controller vendor INDOT personnel became interested in the use of spread spectrum radios to form a multihop network intended to allow control and diagnostic information to be communicated from a location (say a subdistrict) to a remote traffic signal controller. The idea was to replace dialup and/or cellular connections, which are currently used for this purpose. The idea was motivated by radio manufacturer's claims that technology of ISM band spread spectrum radios has improved over the last few years:

- Radio ranges have increased to around 10 to 15 miles under line of sight conditions.

- Previous limitations on the number of repeaters in a network have been eliminated.

The purpose of this part of the project was to investigate the potential to use spread spectrum radios in the manner described above. There were two parts to the work. First, we evaluated the specifications of a particular radio manufactured by Freewave with regard to:

- Receiver sensitivity.

- Link budgets for two propagation models (free space and two-ray models).

- Supported network architectures.

- The effect of repeater operation on throughput.

- The network control requirements needed to operate in a multi-hop scenario.

- Message delay through the network.

Second, we did some field testing with a representative Freewave radio to investigate the practical range in typical Indiana propagation environments with antenna heights corresponding to mounting on top of traffic signal poles and cross arms. 


\section{Analysis of Data}

\subsection{Task A: Support for Field Tests of Satellite Communication Service Providers}

\subsubsection{Satellite Communications Background}

Broadband satellite service has become widely used for broadcasting and for connecting geographically remote sites where terrestrial communications infrastructure is not available. However, there are several characteristics that are unique to satellite communications and can affect the performance, in particular the throughput, when TCP is implemented on a satellite link. The most notable difference between satellite and terrestrial communication is the latency: the typical round-trip delay is $540 \mathrm{~ms}$ for a GEO satellite compared with $100 \mathrm{~ms}$ for a cross-continental Internet connection. Current satellite links also have a large bandwidth-delay product, which implies a large amount of data must be transmitted into the network to fully utilize the capacity of the link. Another problem is the reduced signal-to-noise ratio in satellite communications due to the atmospheric conditions, multipath and shadowing. Measurements show that un-encoded satellite channels can have bit error rates (BER) around $10^{-6}$, much higher than on highspeed wired line. Whenever a packet is lost due to transmission errors, the TCP sender will interpret this as network congestion when in fact there is no congestion. Finally, due to the high cost of ground station equipment, a lower bandwidth is usually provided for the uplink, while higher bandwidth is used for the downlink. The resulting bandwidth asymmetry can lead to acknowledgement (ACK) starvation, in which the TCP acknowledgements flowing back to the sender over the low-speed link arrive too slowly to allow sender fill the satellite link in a timely fashion. All of the above features can cause serious problem for TCP over satellite and has been discussed thoroughly in [Partridge 1997, Metz 1999, Balakrishnan 2001, Muhonen 1998].

\subsubsection{TCP Congestion Control}

To understand the potential problem, a typical TCP congestion-control mechanism is illustrated as follows. This algorithm is due to V. Jacobson [Jacobson 1998] and a number of modifications to Jacobson's initial algorithm are described in Stevens [Stevens 1996] and in RFC 2581 [Allman 1999]. The goal of TCP is to provide fast, reliable endto-end data transmissions. It makes fair and reasonable attempts to fill the link to the extent possible while at the same time avoiding network congestion. To achieve this goal, TCP, in a process called slow-start, carefully probes the capacity of the network in order to let the packets traverse the heterogeneous network seamlessly. Slow-start begins after a connection establishment or a TCP connection time-out, and maintains three variables: CWND, RWND and SSTHRESH. CWND, or the congestion window, imposes a constraint on the maximum amount of data that can be injected into the network before receiving an acknowledgement packet (ACK); RWND, or the receiver window, is 
advertised by the receiver to control the amount of data to be sent to it; SSTHRESH is the ending threshold of the slow-start process. Specifically, the amount of unacknowledged data that the host can have must be always smaller than the minimum of CWND and RWND.

At the beginning of each connection, the congestion window is initialized to one segment and doubled every round-trip time (RTT) until a loss occurs or the congestion window reaches the threshold. Once the congestion window is larger than the current value of SSTHRESH, the slow-start phase ends and the congestion window grows linearly rather than exponentially. This phase of the algorithm is called congestion avoidance. On the other hand, if a packet loss occurs, TCP assumes it was caused by the network congestion and therefore sets SSTHRESH to half of the current congestion window and resets the congestion window to one segment. Then sender must regrow the congestion window exponentially using the slow-start procedure.

TCP also employs a fast retransmission algorithm [Allman 1999] to rapidly recover from a single lost packet, or one that is delivered out of sequence, without shutting down the CWND. When TCP detects the loss of a packet, it acknowledges subsequent packets with the ACK number of the last correctly received packet. When the sender receives three duplicate ACKs, it retransmits the lost packet. The receiver responds with a cumulative ACK for all packets received up to that point. The rationale is that since the subsequent packets were successfully transmitted, it might not be necessary to enter the slow-start phase and reduce the throughput dramatically.

There are several issues with these algorithms when running on a satellite link. Since the duration of the slow-start phase is approximately proportional to RTT $\log _{2}$ RWND, the first problem is that slow-start is even slower in a high latency (RTT) network. In many cases, the entire transfer completes before the slow-start phase ever finishes, and the user never experiences the full bandwidth. Moreover, these mechanisms limit the throughput attainable on a long latency satellite link; in particular, the throughput can be computed by the following equation:

$$
\text { Throughput }=\text { RWND } / \text { RTT } \text {. }
$$

This gives a maximum throughput of $970 \mathrm{kbps}$ given the current standard maximum TCP window size of 65,535 bytes and a roundtrip delay of $540 \mathrm{~ms}$. In other words, no matter how large the bandwidth, the throughput of the standard TCP over a satellite link can never be larger than $970 \mathrm{kbps}$. The TCP window scaling option proposed by RFC 1323 [Jacobson 1992] increases the maximum TCP window size to more than 65,535 bytes. However, changing this parameter requires complex software and network management on every client and server involved. Other than the throughput, it has been indicated [Lakshman 1997] that for multiple connections sharing the same bottleneck link, TCP is grossly unfair toward connections with higher RTT. 


\subsubsection{Alternative Approaches}

A number of approaches have been proposed to alleviate the TCP-over-satellite latency problem. They can be classified into three groups. The first group includes all kinds of TCP modifications or tuning schemes, such as selective acknowledgement (SACK) [Mathis 1996], TCP window scaling [Jacobson 1992], path MTU discovery [Mogul 1990], and others [Ghani 1999, Wang 2004, Samaraweera 1997]. The second approach, is generally referred to as the TCP splitting solution [Henderson 1999, Mhatre 2002, Philopoulos 2002]. An alternative solution to the above two is terminal emulation. We focus on TCP splitting and terminal emulation.

In TCP splitting, the entire end-to-end TCP connection is broken up into three segments by having two proxies placed at each end of the point-to-point satellite link. Then, instead of modifying or tuning the TCP, the proxies terminate the local TCP sessions and map them into a satellite transport protocol (STP) that is suitable for the satellite link. Many satellite service providers have implemented this approach, each in its own (proprietary) way. By implementing the TCP splitting solution, the sender is communicating directly with the near-end proxy instead of the receiver on the far-end. The proxy then converts TCP into a satellite transport protocol, which has window size tuned to the RTT of the satellite link. Moreover, the proxy knows the capacity and the data error rate of the satellite link and hence the slow start procedure is not needed. The net effect is that the sender sees a lower latency network and can keep sending data instead of sending and waiting.

There is yet another approach - terminal emulation - which has the potential to solve the problem by avoiding it entirely. Citrix is such a terminal server system that allows users to access applications from remote locations, regardless of the type of user's computer. All the processes during a Citrix session actually run on the server and not on the user's local machine. The only information transferred between the server and the client is the updated screen shots and user inputs such as mouse movements and keystrokes. In this way the information that needs to be transferred onto the long-delay network is greatly reduced, greatly reducing waiting time.

To determine how well the current satellite network solutions work with applications software of importance in transportation management, an experimental satellite network was set up and a series of tests was carried out as described in the next section.

\subsubsection{The Experimental Network}

Tests were performed using the networks of two satellite service providers, indicated by "A" and "B" in the results to follow. The network topologies were similar in the two cases differing primarily in the location of the service provider's earth station. For brevity we will only describe the network of satellite service provider A in detail as illustrated in Figure 2. The remote office with satellite connection was located in a trailer in West Lafayette, IN. A TCP/IP trace logger was installed on the client test laptop to record the traffic and measure the network performance. At the other end of the satellite link there 
was an earth station (hub) of the service provider in San Diego, CA. The corporate headquarters, wherein all servers resided, was located in Indianapolis, IN, and connected to the service provider through a Virtual Private Network (VPN) over the Internet. As shown in Figure 3, the roundtrip propagation delay for the satellite link is approximately $540 \mathrm{~ms}$ while it is $100 \mathrm{~ms}$ for the Internet connection between San Diego and Indianapolis.

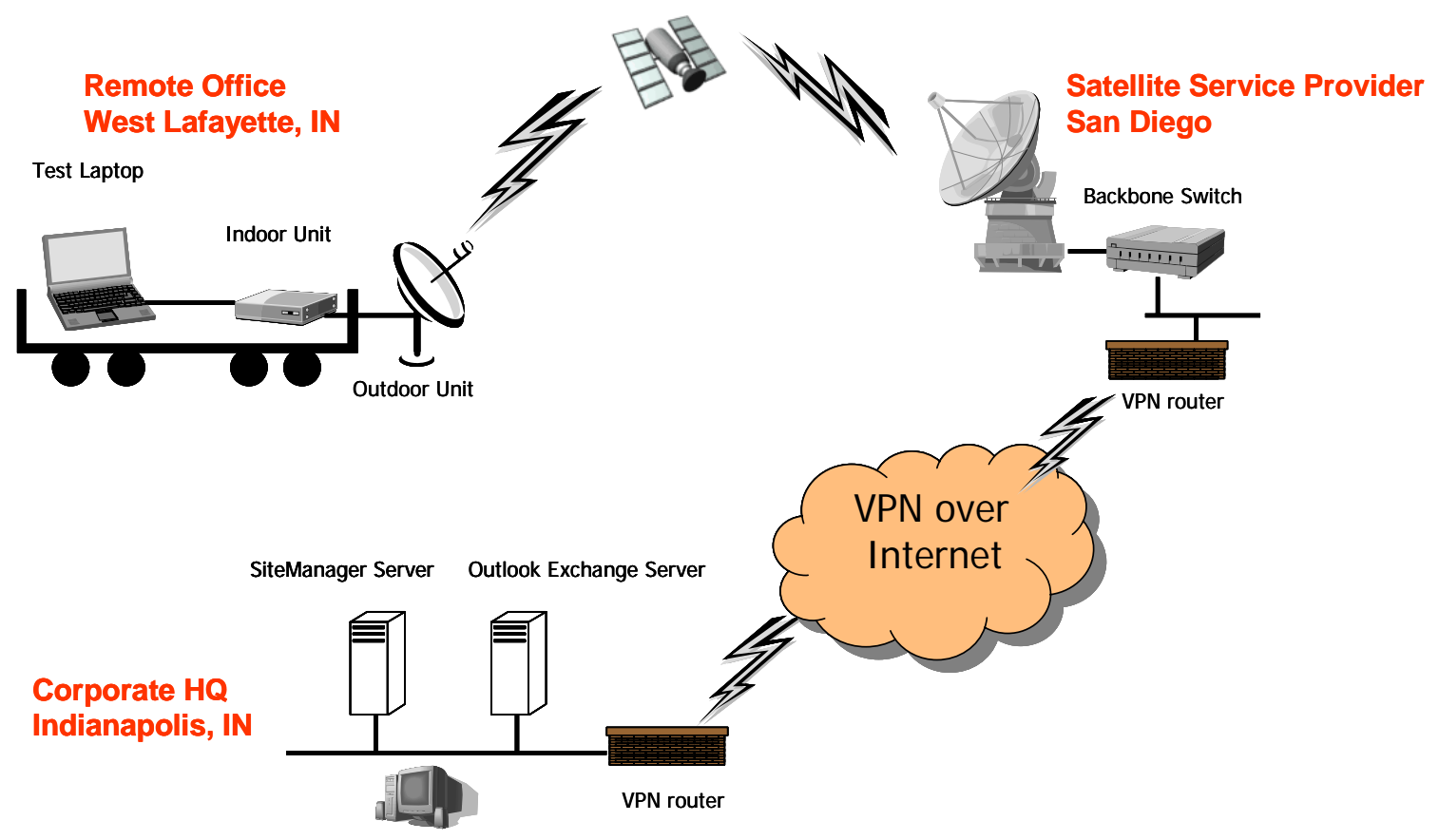

Figure 2: Network topology for service provider A.

The downlink (satellite to remote office) consists of a broadcast channel while the uplink (remote office to satellite) is a multiple-access channel. As is typical in these systems, when the remote terminal needs to initiate communications with the headquarters-based server it first sends a request in the contention-based reservation channel and a time slot is then assigned by the hub in San Diego for subsequent data transmission, i.e., the data transmission is reservation-based. In this system the request itself takes $540 \mathrm{~ms}$ (excluding processing delay at the hub and assuming no collision) and the subsequent data transmission takes $640 \mathrm{~ms}$ (540 ms satellite delay plus $100 \mathrm{~ms}$ Internet delay). Ignoring transmission time for the requested data, the two actions sum to a round trip delay of at least one second from the initial request to the reception of the TCP acknowledgment. Satellite service provider A supports a method to reduce this overhead by sending short messages without reservation. If such a short message goes through without collision then the overall propagation delay has been cut in half. In case a collision occurs, the system just falls back to the reservation scheme. It is obvious that this approach, referred to as the ALOHA mode in the sequel, is most effective when the uplink is not busy. This method is similar in spirit to TCP for Transactions (T/TCP) [Braden 1994] although it should be noted again that the satellite service provider is running its proprietary protocol over the satellite link and not end-to-end TCP. 
Remote Office (West Lafayette)

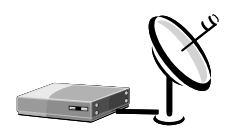

Satellite Service Provider (San Diego)
Corporate HQ (Indianapolis)
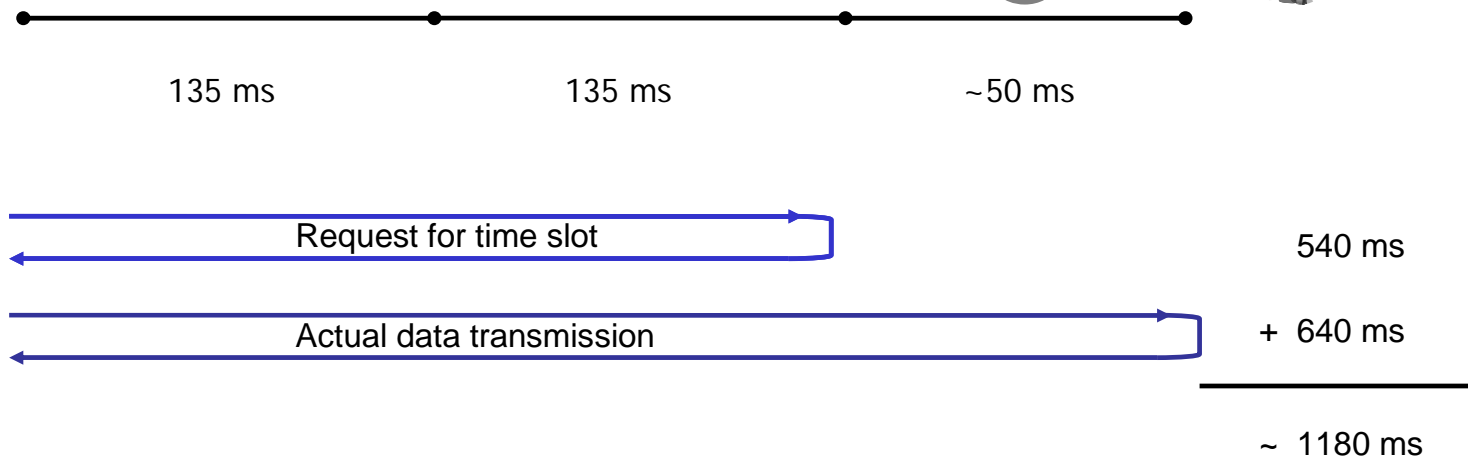

Figure 3: Illustration of components of propagation delay.

\subsubsection{Test Results}

For comparison purposes, two terrestrial tests (without Citrix) were performed: the first with remote offices connected to the headquarters via T1 (1.544Mbps) and the second via dialup (50kbps). A series of satellite tests for the two service providers was then carried out with different bandwidths and operation modes as shown in Table 1.

The applications tested included Microsoft Outlook (O.xx) ( $\mathrm{xx}=$ task number), Windows network drive (E.xx), SiteManager, an Oracle database application intended for use as a comprehensive construction management system (SM.xx), and FTP (FTP.xx). A complete description of each task is listed in Table 2. These tests represent typical construction engineer activities such as moving files to and from remote network drives (E.xx), assembling daily work reports in the AASHTO SiteManager program (SM.xx), and movement of large files to and from a remote server via FTP (FTP.xx). All but a couple of the longest tasks were repeated five times and the average response time to complete each task was computed. Note that the response time depends on three factors: The first is the propagation delay, which is the time it takes for a packet to travel across a link. The second is the transmission delay, which is the time it takes to send a packet onto the network. A 200 byte packet, for example, takes 200 (Bytes) * 8 (bits/byte) / 384 (kbps) $=4$ (ms) to be transmitted onto a 384 kbps satellite link. The last factor is the processing delay (or queuing delay), i.e., the time it takes for servers, clients, or routers to process the data. Processing delay ranges from $1 \mathrm{~ms}$ to several seconds, depending on the protocol and software/hardware involved. 


\begin{tabular}{|c|l|c|}
\hline & \multicolumn{1}{|c|}{ Configuration } & Service Provider Name \\
\hline \multirow{4}{*}{ Terrestrial } & (a) T1 (1.544Mbps) & Level 3 Communications \\
\cline { 2 - 3 } & (b) OC3 (155Mbps) from Purdue with Citrix & I2 \\
\cline { 2 - 3 } & (c) Dialup (50kbps) & AT\&T GlobalNet \\
\cline { 2 - 3 } & (d) Dialup (50kbps) with Citrix & AT\&T GlobalNet \\
\hline \multirow{3}{*}{$\begin{array}{c}\text { Satellite service } \\
\text { provider A }\end{array}$} & (e) 384kbps downlink and 384kbps uplink & Tachyon Network \\
\cline { 2 - 3 } & (f) same as (e) with ALOHA & Tachyon Network \\
\cline { 2 - 3 } & $\begin{array}{l}\text { (g) 1.544Mbps downlink and 512kbps uplink with } \\
\text { ALOHA }\end{array}$ & IsoTropic Networks \\
\hline $\begin{array}{c}\text { Satellite service } \\
\text { provider B }\end{array}$ & (h) 1Mbps downlink and 256kbps uplink & IsoTropic Networks \\
\hline \multirow{4}{*}{\begin{tabular}{c} 
Cellular Wireless \\
\cline { 2 - 3 }
\end{tabular}} & (i) same as (h) with Citrix & Verizon Wireless \\
& \begin{tabular}{l} 
(j) 1xRTT National Access service (PC card for \\
\cline { 2 - 3 }
\end{tabular} & $\begin{array}{l}\text { (k) same as (j) (Motorola V60 phone and Mobile } \\
\text { Office Kit for laptop) with Citrix }\end{array}$ \\
\hline
\end{tabular}

Table 1: Network configurations evaluated.

\subsubsection{T1 terrestrial versus 384kbps/384kbps satellite}

The results for the configuration identified in Table 1, Item (e) (384kbps/384kbps) are shown in Figures 4 and 5. Figure 4a shows the results for shorter tasks (less than 2 minutes) while Figure 4b shows the results for longer tasks. In Figure 4a and 4b the expected times are obtained by scaling the T1 results by a factor equal to the ratio of the satellite bandwidth to the T1 bandwidth. For example, the ratio of T1 bandwidth (1.544 Mbps) to $384 \mathrm{kbps}$ satellite bandwidth is 4, hence the expected time for each task done on the $384 \mathrm{kbps}$ / $384 \mathrm{kbps}$ link should be four times of the corresponding task observed on a T1 link. Note that the above computation only reflects the effect of transmission delay, i.e., the bandwidth. In other words, it is assumed that the response time comes solely from the transmission of data. Since the computation of the expected time doesn't take the propagation delay into account, the observed time for the satellite tests are in general worse than the expected time. The only exceptions are some database procedures (SM.12 and SM.13) where there is minimal data transmission and the main time contribution comes from the server. Since the server processing time does not depend on the network environment the performance for these two tasks is not heavily effected. Figure 5 shows the ratio of the response time on the $384 \mathrm{kbps} / 384 \mathrm{kbps}$ satellite link to that of the terrestrial T1 link. The horizontal line indicates the expected ratio, which is 4 . It can be seen that while other applications have performances close to the $4: 1$ ratio line shown in the figures, most of the SiteManager exercises (SM.xx) tested had poorer performance (higher ratio). 


\begin{tabular}{|c|c|}
\hline $\begin{array}{l}\text { Task } \\
\text { Number }\end{array}$ & Description of the task \\
\hline O.01 & Incoming mail with a 900KB attachment \\
\hline O.02 & Outgoing mail with a 900KB attachment \\
\hline $\mathrm{O} .03$ & Incoming mail with a 7.66MB attachment \\
\hline $\mathrm{O} .04$ & Outgoing mail with a 7.66MB attachment \\
\hline O.05 & Calendar / Jan 20 / double click 12 am \\
\hline 0.06 & $\begin{array}{l}\text { Calendar / Jan } 20 \text { / double click } 12 \text { am / Subject: test, Content: the first two pages from } \\
\text { test.doc / Close and Save }\end{array}$ \\
\hline 0.07 & Public Folders / All Public Folders / Agency Information / Double click Vendors Contact List \\
\hline O.08 & $\begin{array}{l}\text { Public Folders / All Public Folders / Agency Information / Vendors Contact List / Double } \\
\text { click Burns \& McDonnell }\end{array}$ \\
\hline E.01 & $\begin{array}{l}\text { Copy Ilindotrs4lsmgr|Training_Photos_030407\DSCN0465.JPG (900KB) and paste it onto } \\
\text { desktop }\end{array}$ \\
\hline E.02 & Copy DSCN0465.JPG (900KB) to \lindotrs4lsmgr|BWTest \\
\hline E.03 & Copy \lindotrs4lsmgr/smdbl00.db (6608 KB) and paste it onto desktop \\
\hline E.04 & Copy smdbl00.db (6608 KB) to \indotrs4\smgr|BWTest \\
\hline E.05 & Copy TESTFILE.zip (763330 KB) from \lindotrs4\smgrlBWTest and paste it onto desktop \\
\hline E.06 & Copy TESTFILE.zip (763330 KB) to \lindotrs4|smgr|BWTest \\
\hline FTP.01 & Download bigfile.dat (100000 KB) \\
\hline FTP.02 & Upload bigfile.dat (100000 KB) \\
\hline SM.01 & Run SiteManager / Server Mode / User name and password \\
\hline SM.02 & $\begin{array}{l}\text { Accessories / In-Box / Service / Compose / To: Chen, T.C. / Subject: Test / Message: copy } \\
\text { and paste the first two pages from test.doc / Services / Send }\end{array}$ \\
\hline SM.03 & Accessories / In-Box / double click the mail sent in procedure SM.02 \\
\hline SM.04 & $\begin{array}{l}\text { Pipeline and Zip / server to PM pipeline / open Contract R-26013-G / Check Basic Contract } \\
\text { Data -> Service -> PipelineData }\end{array}$ \\
\hline SM.05 & $\begin{array}{l}\text { Pipeline and Zip / PM to Server Pipeline / open Contract R-26013-G / Check Basic Contract } \\
\text { Data / Service / PipelineData }\end{array}$ \\
\hline SM.06 & $\begin{array}{l}\text { Change orders / Change order Maintenance / Change Order Item / New Contract Item tab / } \\
\text { Right click item code to do a search }\end{array}$ \\
\hline SM.07 & Daily Work Report / Daily Work Report \\
\hline SM.08 & Daily Work Report / Daily Work Report / Change from Info view to Contractor view \\
\hline SM.09 & Daily Work Report / Daily Work Report / Change from Daily Staff view to Work Items view \\
\hline SM.10 & $\begin{array}{l}\text { Daily Work Report / Daily Work Report / Double click Construction Engineering under Work } \\
\text { Items view }\end{array}$ \\
\hline SM.11 & Daily Work Reports / Diary / Check Authorized / Save \\
\hline SM.12 & Contractor Payments / Estimate / Generate Estimate / Services / Generate Estimate \\
\hline SM.13 & $\begin{array}{l}\text { Contractor Payments / Process List / Select R-26013-G and double click to submit (clock } \\
\text { starts now) / Process Submitted click ok / Process Completed }\end{array}$ \\
\hline SM.14 & $\begin{array}{l}\text { Contractor Payments / Reports / Summary by Project / Double Click R-26013-G / Select } \\
\text { Estimate Nbr } 0001\end{array}$ \\
\hline
\end{tabular}

Table 2: Description of exercises conducted with Outlook, Explorer, FTP, and SiteManager. 


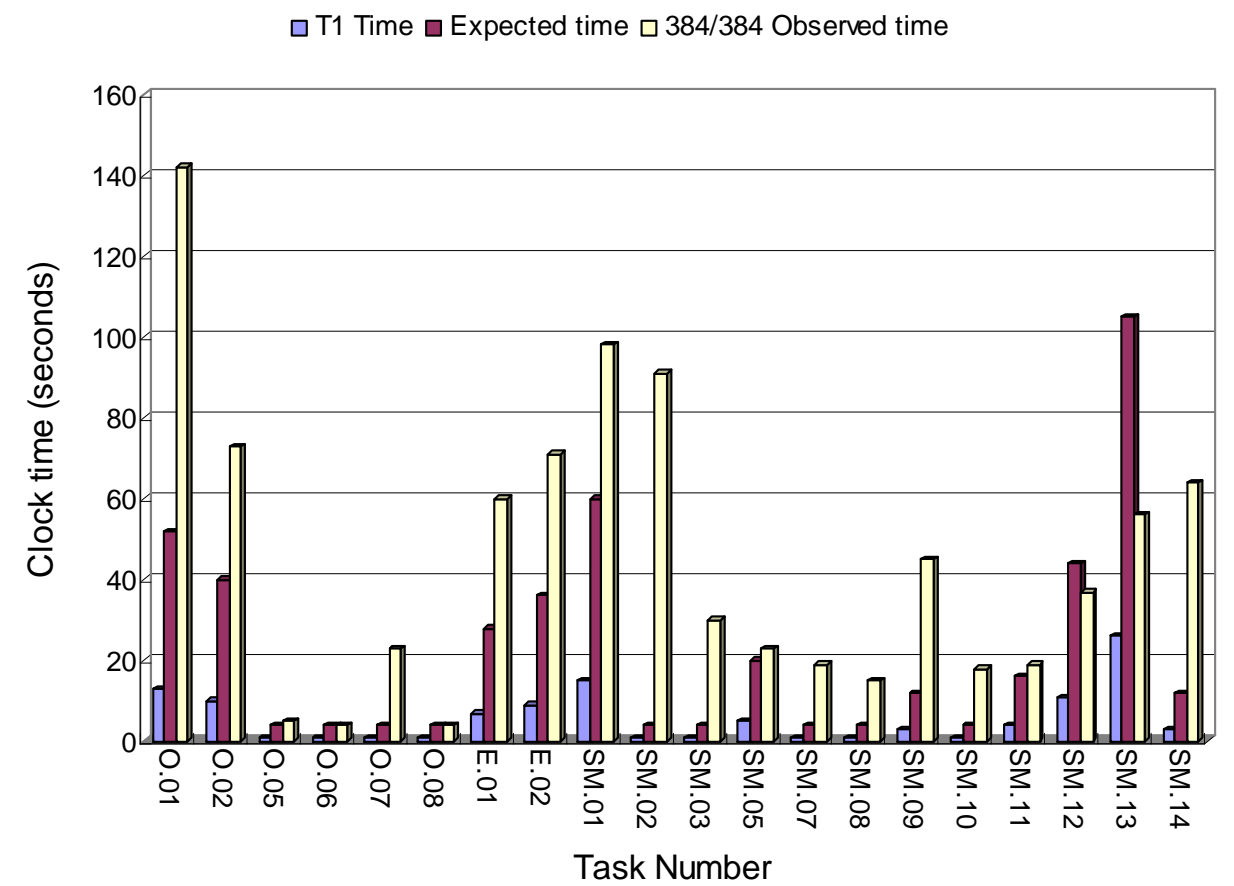

a) Performances for $384 \mathrm{kbps} / 384 \mathrm{kbps}$ (shorter tasks).

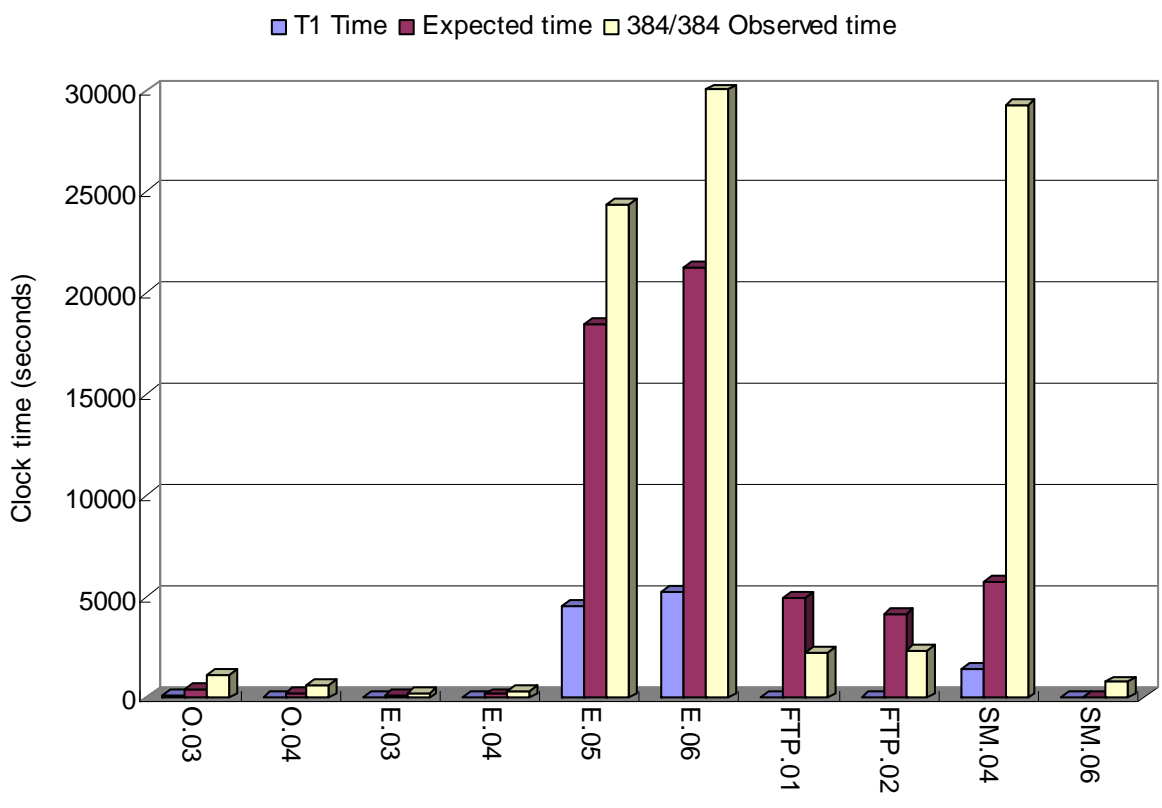

Task Number

b) Performances for 384kbps/384kbps (longer tasks).

Figure 4: T1 terrestrial versus 384 kbps / 384 kbps satellite. 


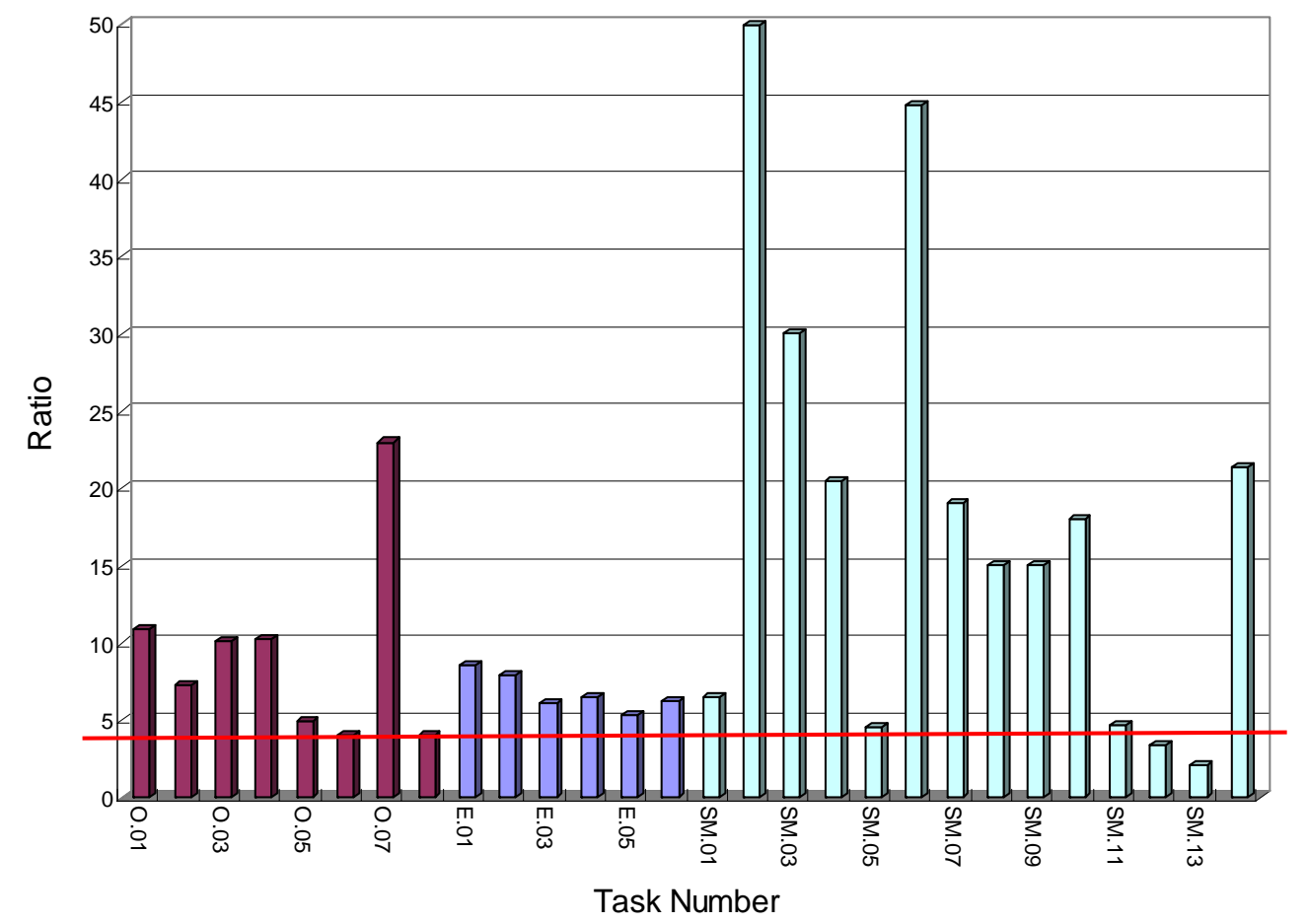

Figure 5: Ratio of 384 kbps / 384 kbps response time to T1 response time.

\subsubsection{Non-ALOHA versus ALOHA}

Comparing configurations (Table 1, Item (e)) and (Table 1, Item (f)) the only difference is whether or not the ALOHA option is enabled. To reduce the chance of collision, only packets with sizes less than 200 bytes are allowed to be sent via ALOHA. The number is selected after examining the packets of SM.xx tasks where a large portion of the packets are of length less than 200. As can be seen in Figure 6 where the first column shows the normal mode and the second column the ALOHA mode, enabling ALOHA results in an improvement of $18 \%$ on average. Since ALOHA was observed to enhance the performance for every task, it was used for the remaining tests on service provider A.

\subsubsection{384kbps/384kbps versus $1.544 M b p s / 512 k b p s$}

Response time comparisons between configurations options defined in Table 1, Items (e), (f) and (g) are shown in Figure 7. It can be seen that, on average there is only about $10 \%$ improvement when the bandwidth is increased from $384 \mathrm{kbps} / 384 \mathrm{kbps}$ to $1.544 \mathrm{Mbps} /$ 512 kbps (both with ALOHA mode enabled). This illustrates that increasing bandwidth on a satellite link has a negligible impact when an application, such as SiteManager is chatty, and there are long communication latencies. 


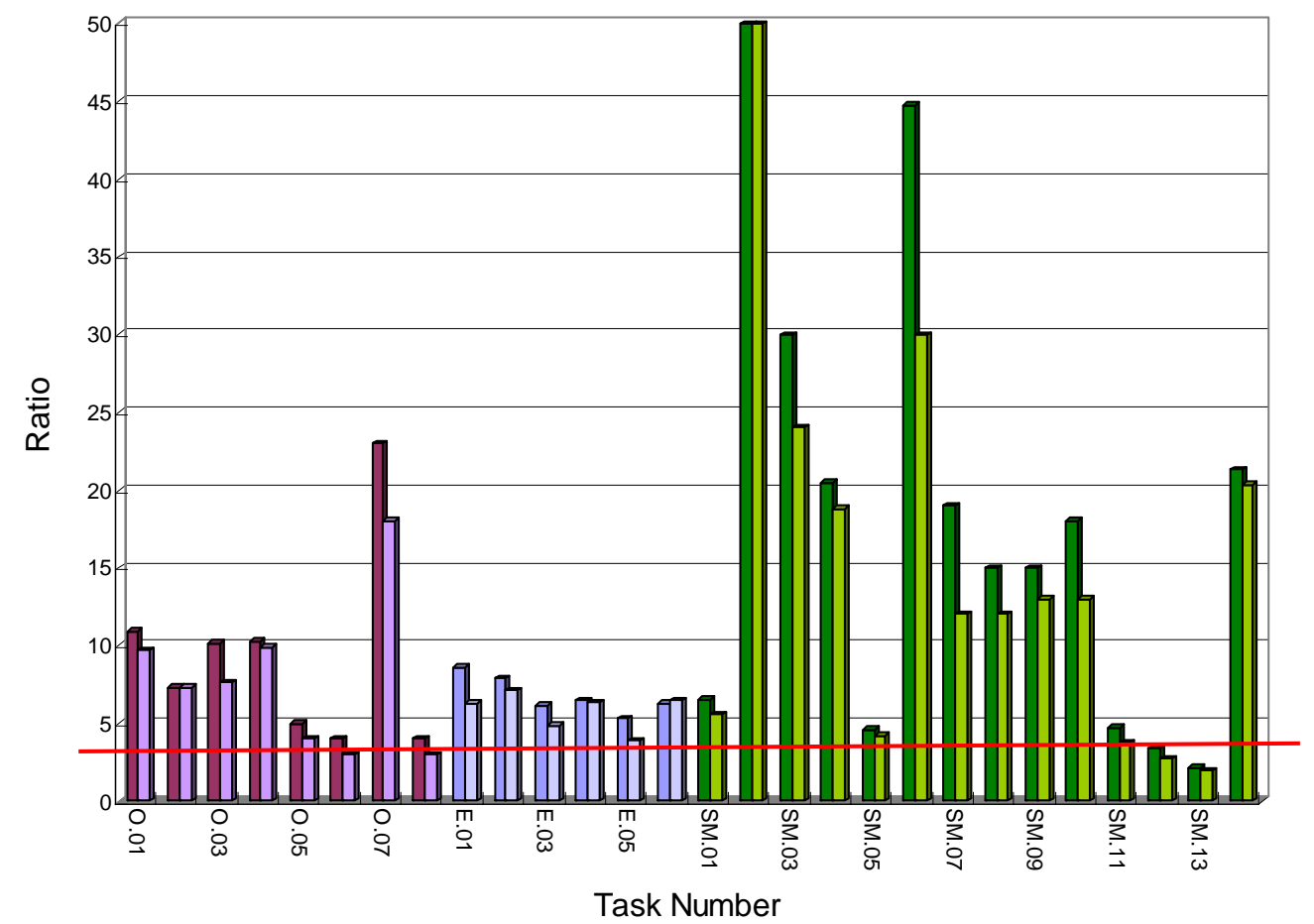

Figure 6: Ratios of 384 kbps / 384 kbps (first column) and 384 kbps / 384 kbps with ALOHA (second column) to T1 response time.

\subsubsection{T1 versus 384/384 ALOHA versus Dialup}

To provide a comparison between dialup terrestrial service and satellite communication, the configurations shown in Table 1, Items (a), (c), and (f) are compared to document the effect that different propagation delays and transmission delays (bandwidth) have on performance. The T1 link has both low propagation and transmission delays, the satellite link has high propagation delay and median transmission delay, and the dialup link has low propagation delay but high transmission delay. From Figure 8 it can be seen that for data transmission-intensive applications such as O.01, O.02, E.01, and E.02, larger bandwidth provides significantly better performance, while for most of the database tasks, it is the low propagation delay, rather than the larger bandwidth, that improves performance.

From the above tests it can be seen that the performances of many applications are not bandwidth limited. In fact, by computing the effective throughput as shown in Figure 9, it can be seen that, while the throughput approaches the provided bandwidth for FTP tasks, the database programs we tested were not efficient users of bandwidth. To further explore this phenomenon the TCP trace was recorded for SM.07, a procedure that creates a blank form on the client. As shown in Figure 10 where the blue lines represent outgoing traffic 


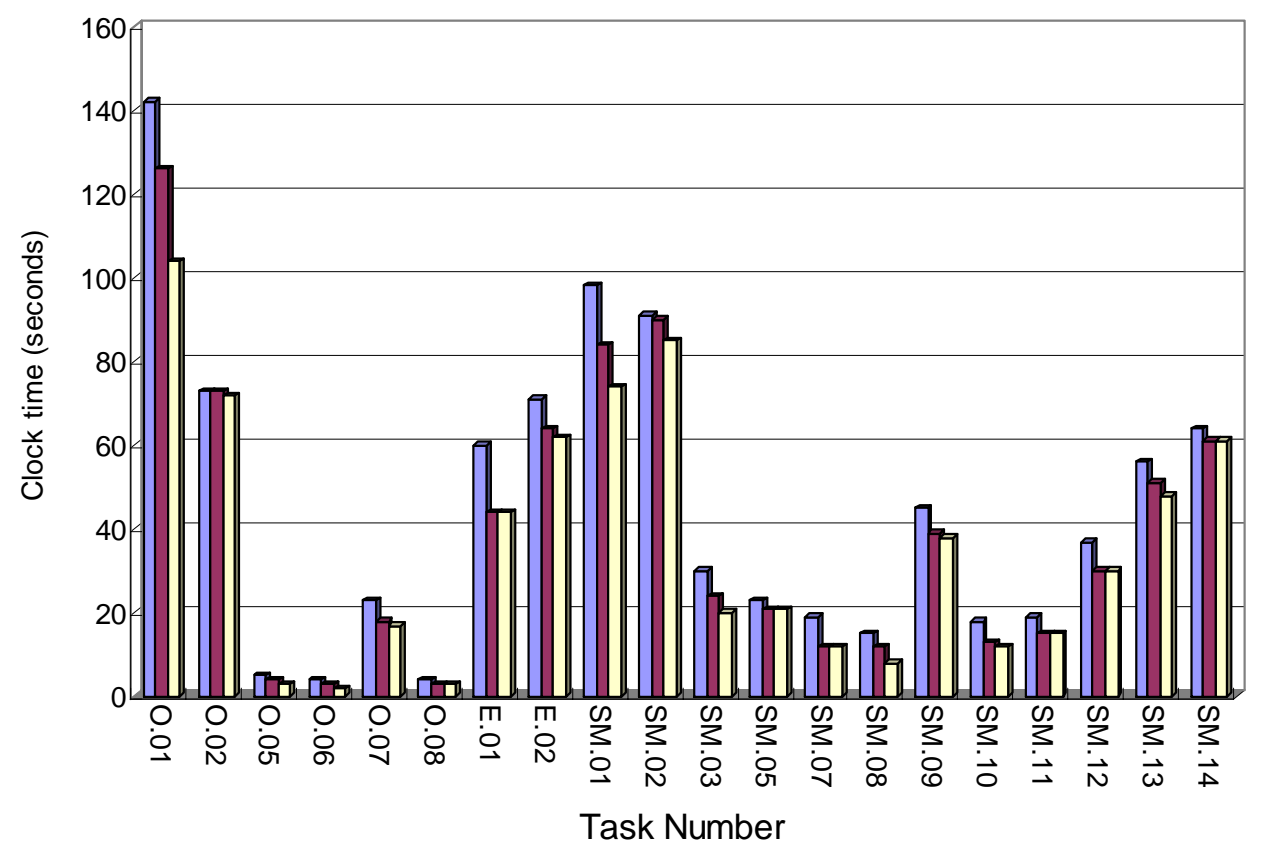

a) Performances of 1.544 Mbps / 512 kbps with ALOHA (shorter tasks)

$\square$ 384/384 Observed time $\square$ 384/384 W/ALOHA Observed time $\square$ 1544/512 w/ALOHA Observed time

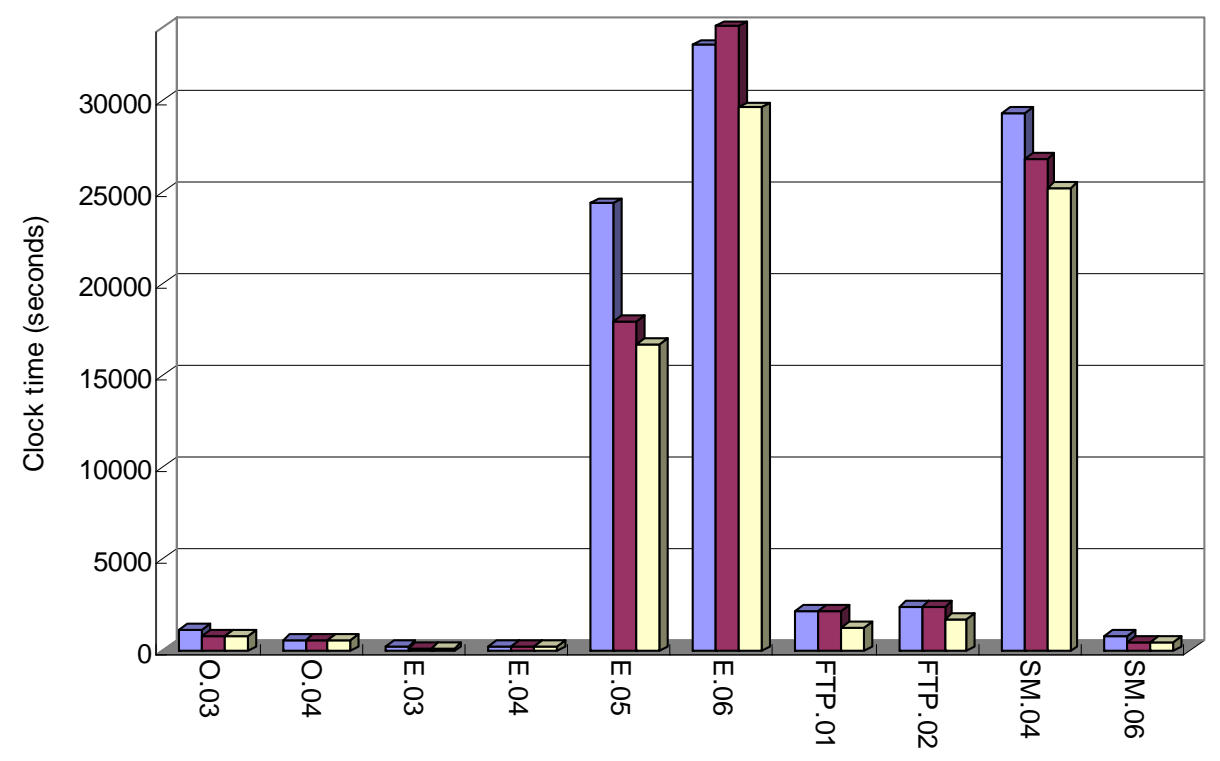

Task Number

b) Performances of 1.544 Mbps / 512 kbps with ALOHA (longer tasks)

Figure 7: 384 kbps / 384 kbps versus 1.544 Mbps / 512 kbps. 
$\square$ T1 Time $\square$ 384/384 w/ALOHA observed time $\square$ Dialup Time

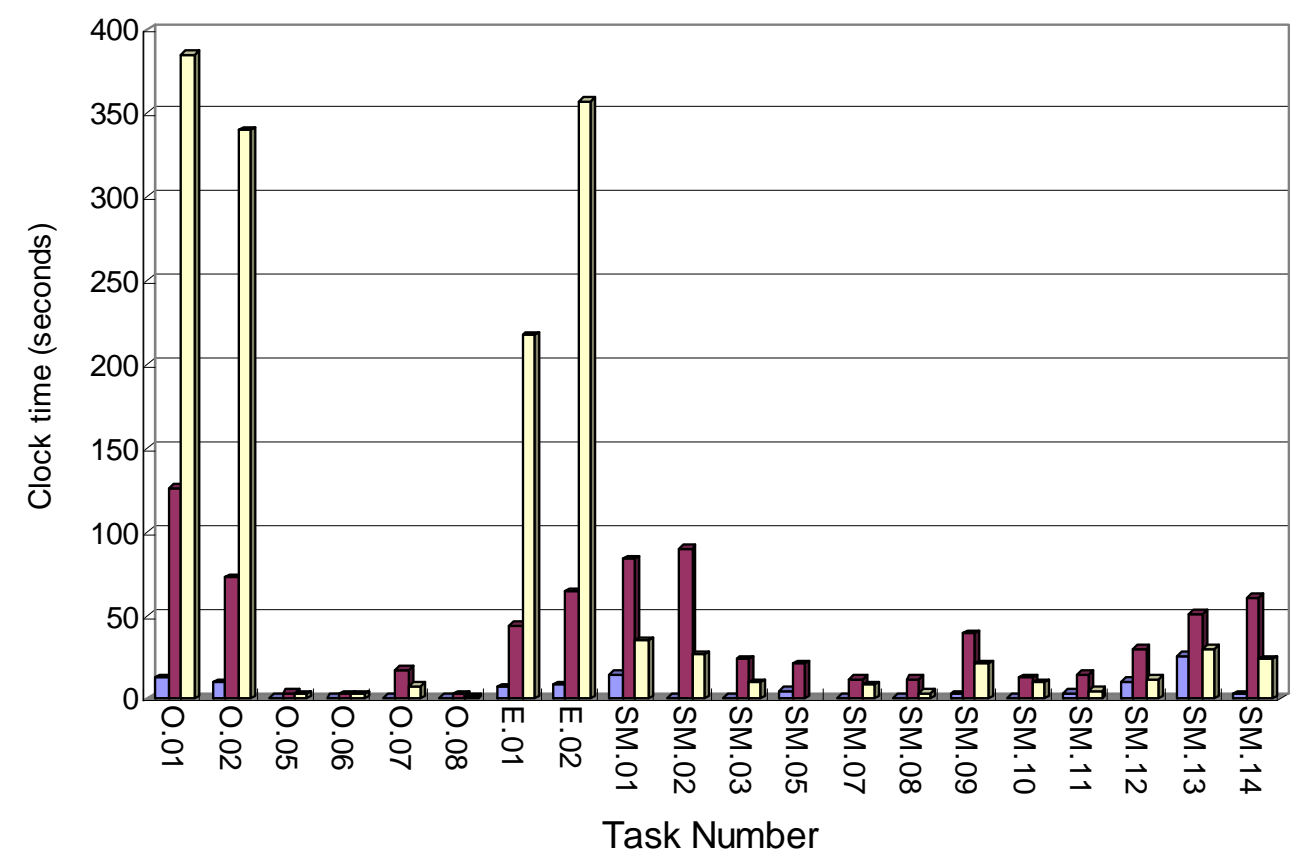

Figure 8: Performances of T1 versus 384 kbps / 384 kbps ALOHA versus dialup.

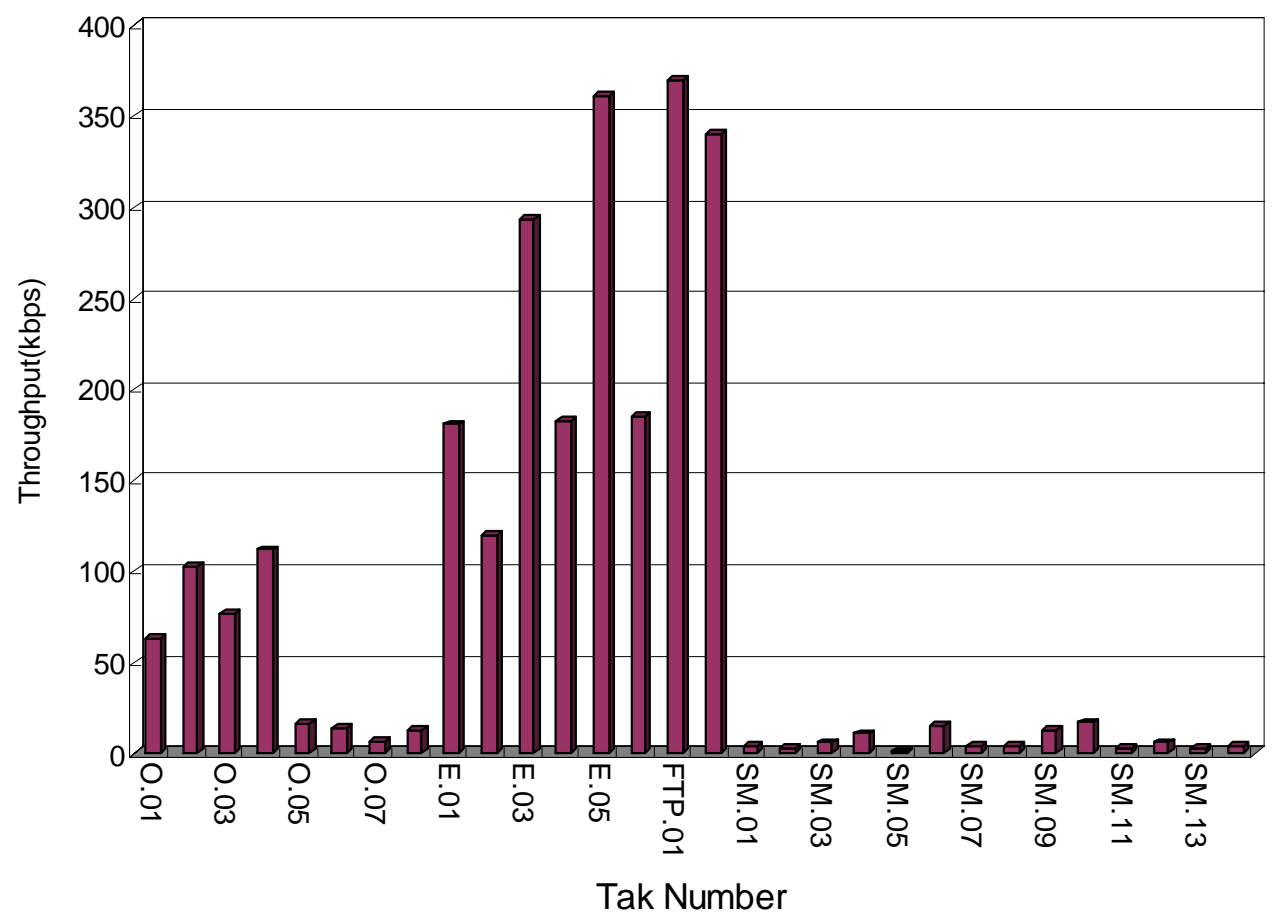

Figure 9: Throughput observed for 384 kbps / 384 kbps with ALOHA. 


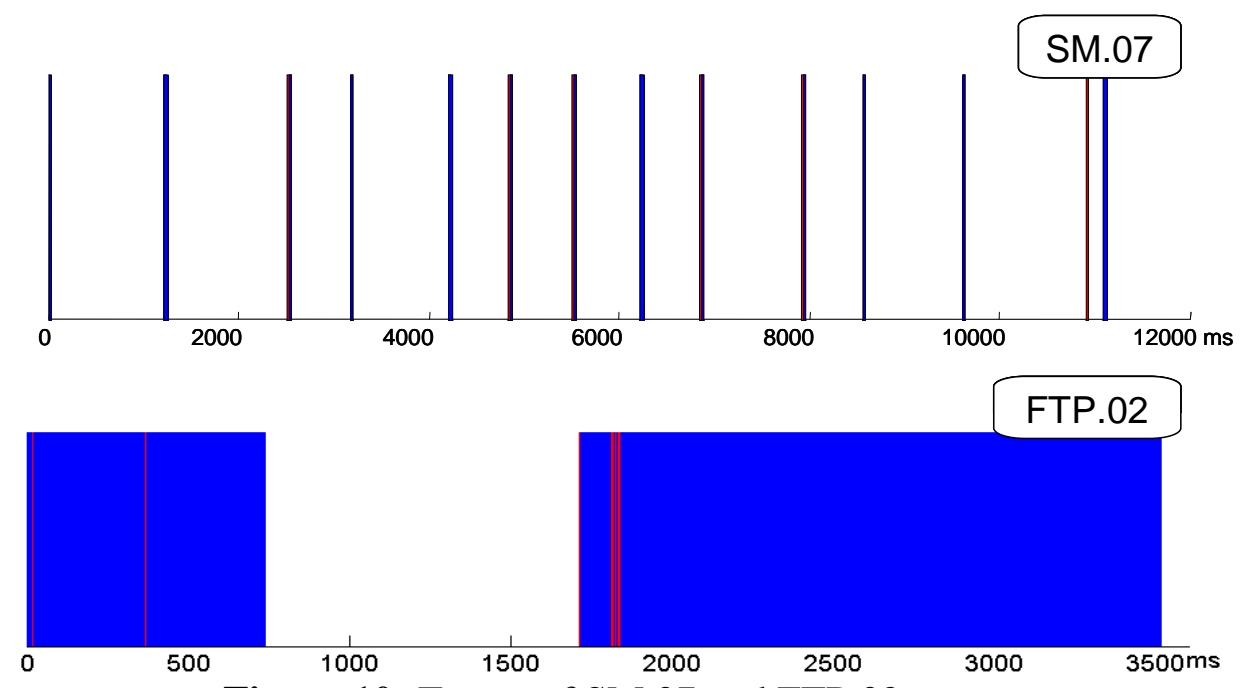

Figure 10: Traces of SM.07 and FTP.02.

(from client) and the red lines represent incoming traffic, the client spends most of the time waiting when SM.07 is executed. In contrast, more than $75 \%$ of the time is dedicated to transmission when FTP.02 is executed. To be more specific, application SM.07 consists of 12 request/response pairs executed sequentially, i.e., in order to send a new request, the client has to wait until it receives the response from previous request. Moreover, it was found that the responding packets in the database program are not "pure" TCP acknowledgments but instead carry other information. The sender therefore keeps waiting until the response, which contains the necessary information is received. On the other hand, the acknowledgment generated by the proxy does not contain the information needed and is ignored. As a consequence, the TCP acceleration is ineffective for SM.xx applications.

Knowing the logic and timing structure of each database procedure, the response time can actually be predicted. Take SM.07 for example. Let $D$ be the total amount of data to be sent and received, $N_{1}$ and $T_{1}$ be the number of packets sent via ALOHA (i.e., packets less than 200 bytes) and the corresponding propagation delay, respectively, $N_{2}$ and $T_{2}$ be the number of packets sent without ALOHA and the corresponding propagation delay, $T_{\text {proc }}$ be the total processing time spent at client and server, and $B$ be the bandwidth provided, the response time $T$ can be approximated by

$$
T=\left(N_{1} T_{1}+N_{2} T_{2}\right)+D / B+T_{p r o c}
$$

where in the above equation the first term and the second term accounts for the propagation delay and the transmission delay, respectively. Figure 11 shows the predicted response time for SM.07 under different provided bandwidth. It is again apparent that increasing bandwidth $B$ brings almost no improvement because the main time contribution comes from large $N$ and $T$. While the propagation delay $T$ can't be altered, $N_{1}$ and $N_{2}$ can be reduced by rewriting the application code so that multiple requests can be accumulated and sent all at once. 


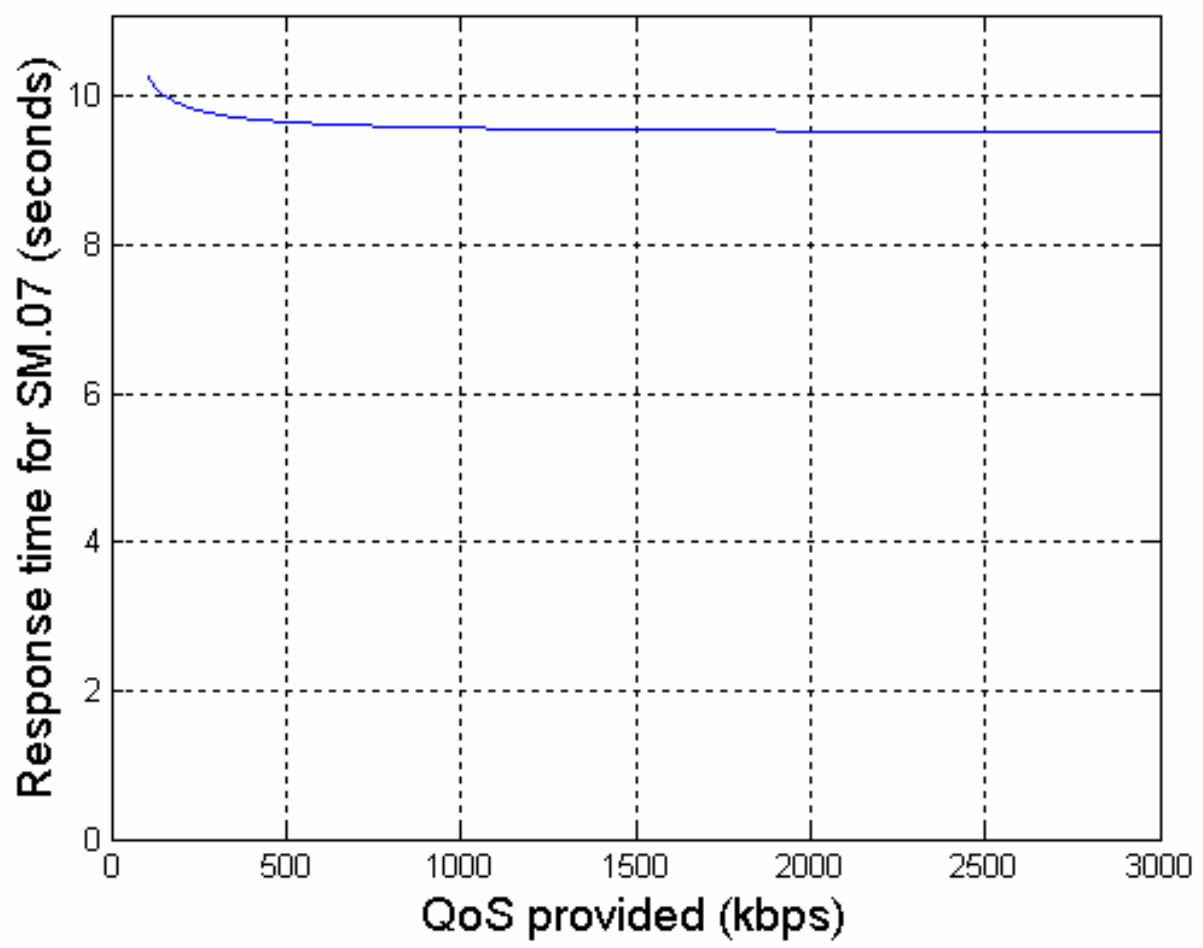

Figure 11: Predicted response time for SM.07 as bandwidth (QoS) provided varies.

\subsubsection{Service provider A versus $B$}

Configurations shown in Table 1, Items (f), (g), and (h) are compared to confirm that the poor performance of the database application is not a consequence of the particular acceleration protocol. A subset of tasks were performed and the results in Figure 12 show that, in general, service provider B has a slightly better performance for the database application, but the throughput is still very low compared to the bandwidth provided. The reason that service provider $B$ has a slightly better performance can be explained by the multiple access scheme it uses, called Deterministic TDMA (D-TDMA). The idea of DTDMA is that each remote office is permanently assigned a time slot (with bandwidth generally less than $8 \mathrm{kbps}$ ). Additional bandwidth is then dynamically allocated by the hub and hence no reservation is required. This effectively reduces the total response time but still the 12 requests/responses are observed sequentially and contribute to a large portion of the total response time.

\subsubsection{Remote Terminal Emulation}

As the particular database program we tested is not designed for the long latency network and hence can't be accelerated, the only way to boost the performance is to use a remote terminal emulation program where only user inputs and screen shots are transmitted and 
all other data are exchanged locally on the server side. Here we set up a series of tests using Citrix with five different connections, namely, Table 1, Items (b), (d), (i), (j), and (k). In Items (j) and (k), 1xRTT is short for single carrier radio transmission technology, which also referred to as cdma2000, is a 3G wireless technology based on the CDMA platform. The Verizon 1xRTT National Access service advertises an average rate of 60 to $80 \mathrm{kbps}$ with bursts up to $144 \mathrm{bps}$. It covers more than $80 \%$ of the state of Indiana (see Figure 1). Note that the configurations in Items (j) and (k) use the same service except that in (j) a PC card is used while in (k) a mobile phone is used. They have different antenna configurations/placements and hence the measured throughputs may differ.

As shown in Figure 13, the performance of using Citrix is better than T1 connection (without Citrix) since most of the data exchanges occur locally on server's local area network. Also note that the clock times are almost the same for tasks running on Citrix regardless what the underlying connection is. That is, dialup service has essentially the same response time as the broadband satellite when Citrix is used. Since occasionally it's more convenient to have a local replica of the files, we also test these connections without Citrix. Table 3 and Figure 14 show the measured throughput and response time respectively when files are actually moved between remote desktop and central office.

One minor issue with Citrix is the color palette used by the server - it may not use the highest color quality (32bits) for display and hence fidelity problem could arise.

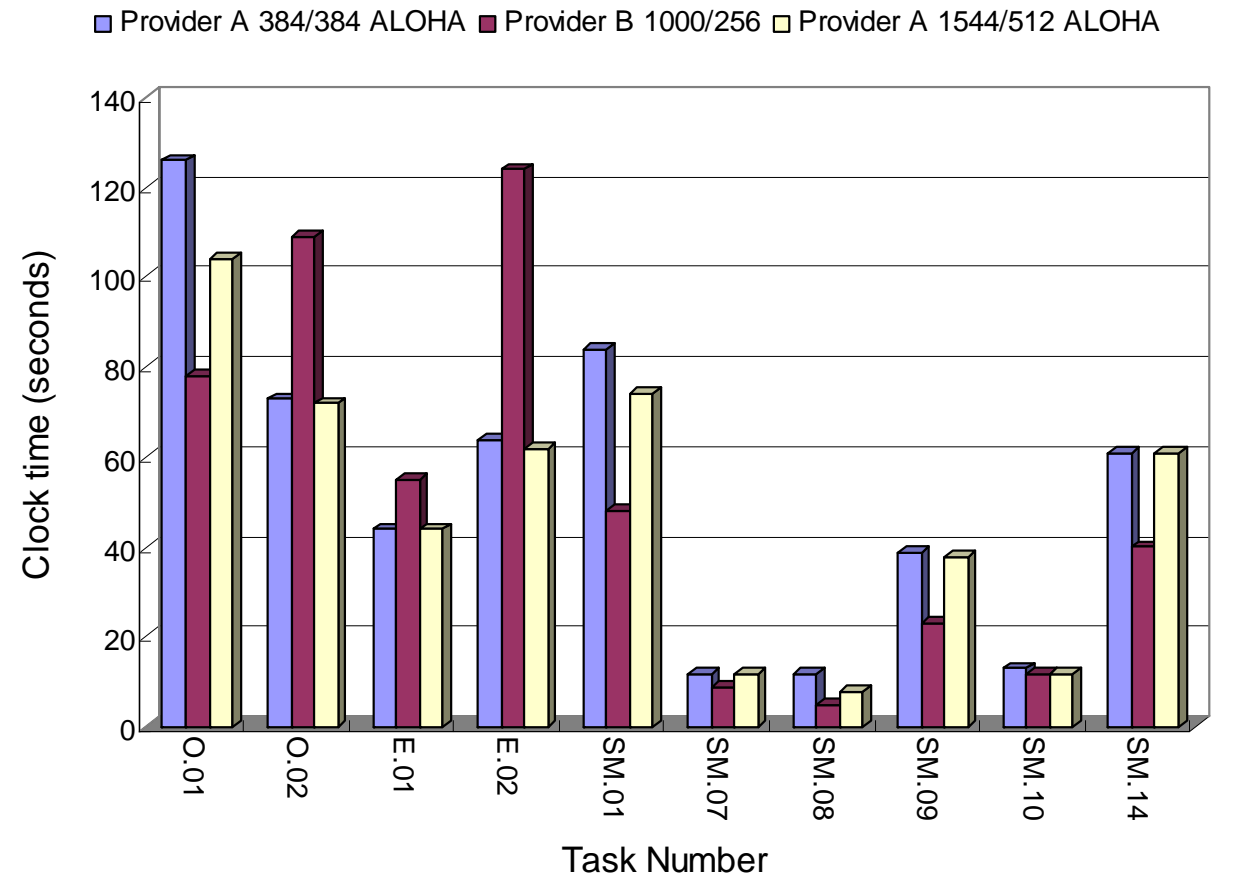

Figure 12: Service provider A versus service provider B. 


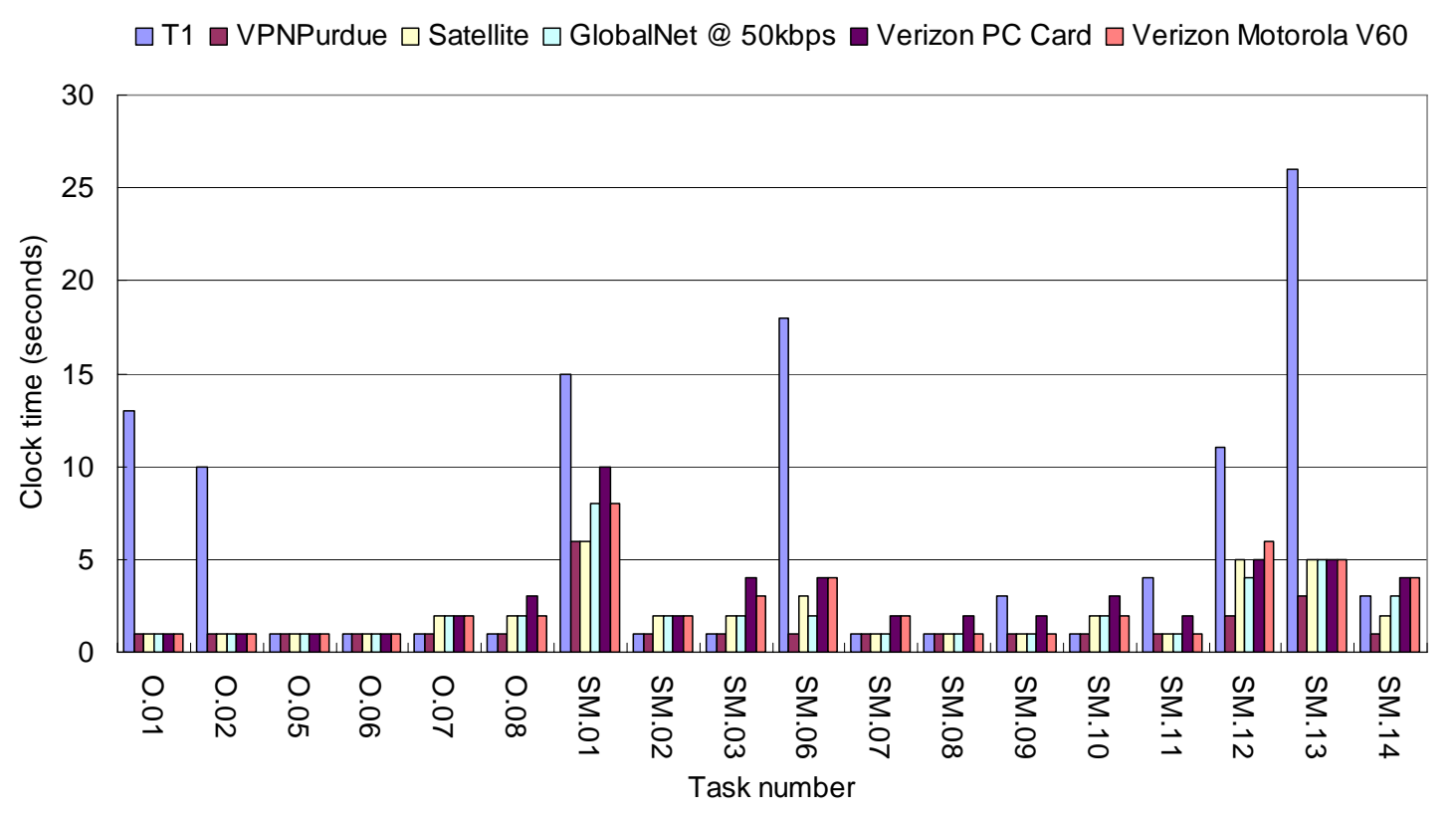

Figure 13: Performances for terminal emulation.

\begin{tabular}{|l|l|l|l|l|l|l|}
\hline $\begin{array}{l}\text { Connection } \\
\text { type }\end{array}$ & T1 & $\begin{array}{l}\text { VPN } \\
\text { Purdue }\end{array}$ & Satellite & $\begin{array}{l}\text { GlobalNet } \\
\text { at 50kbps }\end{array}$ & $\begin{array}{l}\text { Verizon } \\
\text { PC Card }\end{array}$ & $\begin{array}{l}\text { Verizon } \\
\text { Motorola v60 }\end{array}$ \\
\hline $\begin{array}{l}\text { Measured } \\
\text { throughput }\end{array}$ & $\sim 1.4 \mathrm{Mbps}$ & $\sim 20 \mathrm{Mbps}$ & $\sim 100 \mathrm{kbps}$ & $\sim 27 \mathrm{kbps}$ & $\sim 75 \mathrm{kbps}$ & $\sim 58 \mathrm{kbps}$ \\
\hline
\end{tabular}

Table 3: Measured throughputs for different connections.

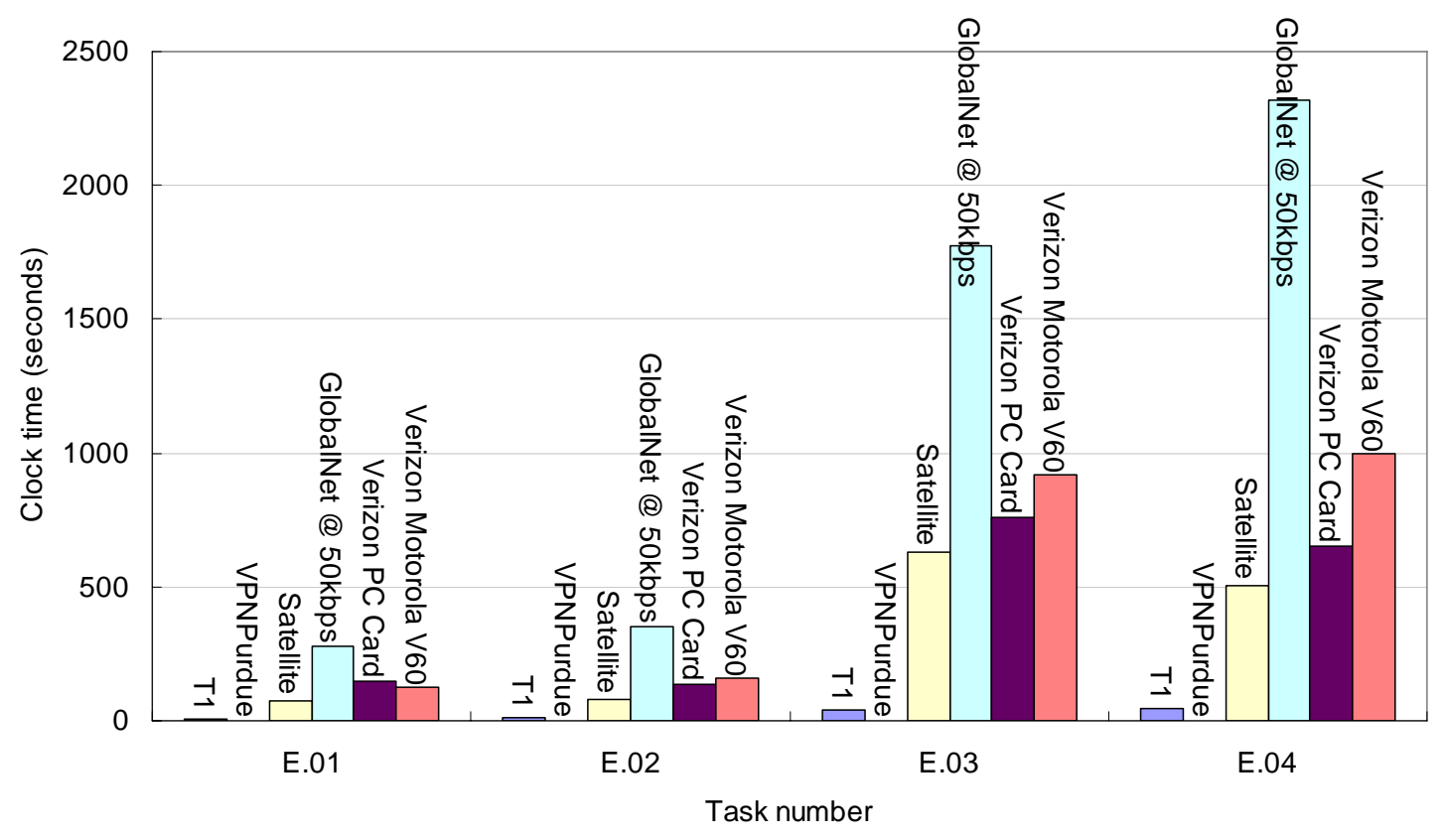

Figure 14: Performance for non-Citrix applications using different connections. 


\subsection{Task B: Spread Spectrum Radio Testing}

\subsubsection{Summary of Specifications of Tested Radio}

The radio tested was the Freewave FGR $900 \mathrm{MHz}$ series frequency-hopped spread spectrum radio. It operates in the ISM band at 902 to $928 \mathrm{MHz}$ and can transmit output power between $1 \mathrm{~mW}$ and $1 \mathrm{~W}$. The radio is frequency-hopped and uses Gaussian frequency shift keying (GFSK) as its modulation method. The modulation occupies an instantaneous bandwidth of $230 \mathrm{kHz}$. It provides a link throughput between 38.4 and 155 kbps and operates with receiver sensitivity between -111 and $-108 \mathrm{dBm}$.

\subsubsection{Theoretical Range Calculation from Specifications and Propagation Modeling}

\subsubsection{Receiver Sensitivity}

This is the first key parameter that is used to characterize the performance of a radio system. Receiver sensitivity is defined as the minimum signal threshold that can be detected in the presence of noise. It is calculated as follows (all quantities below are expressed in $\mathrm{dB}$ ):

$S=K T B+$ SNR to achieve target BER $+N F$

where $S$ is the receiver sensitivity, $k$ is Boltzmann's constant, $T$ is the temperature in Kelvin, $B$ is the receiver bandwidth, and $N F$ is the receiver's noise figure.

When doing a link budget analysis, it is important to design the system such that the received signal strength is larger that the receiver sensitivity.

\subsubsection{Thermal Noise}

The receiver antenna introduces thermal noise caused by the random motion of electrons into the system. Thermal noise power is defined as,

$P_{n}=k T_{o} B$

where $k$ is Boltzmann's constant given by $1.38 \times 10^{-23}$ joules/Kelvin and $B$ is the equivalent bandwidth. For the Freewave $900 \mathrm{MHz}$ radios $B$ is $230 \mathrm{kHz}$. $T_{o}$ is the ambient room temperature and is typically around $290 \mathrm{~K}$ ( $27^{\circ} \mathrm{C}$ ). Thermal noise introduced at at the Freewave front-end is around:

$$
\left(P_{n}\right)_{d B m}=10 \log _{10}\left(k T_{o} B \times 1000\right)=10 \log _{10}\left(1.38 \times 10^{-23} \times 290 \times 230,000 \times 1000\right)=-120 d B m
$$




\subsubsection{SNR to Achieve a Target BER}

A Golay $(24,12)$ code typically requires an $E_{b} / N_{o}$ of $6 \mathrm{~dB}$ to achieve a BER of $10^{-6}$ for antipodal signaling [Hackett 1981]. This is approximately what would be required by the Freewave system which uses GFSK with a retransmit on error scheme. Therefore, if the bandwidth occupancy ( $W$ ) is $230 \mathrm{kHz}$ with a data rate $(R)$ of $170 \mathrm{kbps}$, the required SNR to achieve a BER of $10^{-6}$ is:

$$
\left(\frac{S}{N}\right)_{d B}=10 \log _{10}\left(\frac{R}{W} \cdot \frac{E_{b}}{N_{o}}\right)=10 \log _{10}\left(\frac{170}{230} \cdot 10^{0.6}\right)=5 d B
$$

\subsubsection{Noise Figure}

The receiver chain, i.e., all stages from the front end of the receiver to the back end also generates noise. Noise Figure is a figure of merit for how noisy the receiver chain is. It is defined as:

$$
N F=\frac{\text { Noise output of actual receiver }}{\text { Noise output of ideal receiver }}
$$

Typical values of $N F$ range from 4 to $15 \mathrm{~dB}$. Given that the receiver sensitivity for the Freewave radio is around $-111 \mathrm{dBm}$ for a BER of $10^{-6}$ at low speed then the Noise Figure can be estimated to be around:

$$
N F=-111+120-5=4 d b
$$

\subsubsection{Link Budget Analysis for Free Space Propagation}

Assuming omnidirectional receive and transmit antenna's Frii's equation gives us the path loss in free space:

$$
L(d B)=20 \log _{10}\left(\frac{4 \pi d}{\lambda}\right)
$$

where $\mathrm{d}$ is the distance between transmit and receive antennas and $\lambda$ is the wavelength of the transmitted signal (at $900 \mathrm{MHz} \quad \lambda=0.33 \mathrm{~m}$ ) . The above expression does not include the effects of multipath and large/small signal fading.

The Freewave manual specifies that the maximum range using unity gain antennas is 60 miles. From the equation above this implies that $L(d B)=131 d B$. For a receiver 
sensitivity of $-113 \mathrm{dBm}$ and with the maximum output transmitter power being $30 \mathrm{dBm}$, the losses due to antenna cables, etc., are:

$P_{\text {loss }}=30-131+113=12 d B$

We can now calculate the radio ranges for a more realistic environment.

\subsubsection{Link Budget Analysis for a Two-Ray Model and Fading}

The two-ray model is a more realistic model compared to the free space propagation model. With the two-ray model there is both a direct path and a ground reflection propagation path between the transmitter and receiver as shown below:

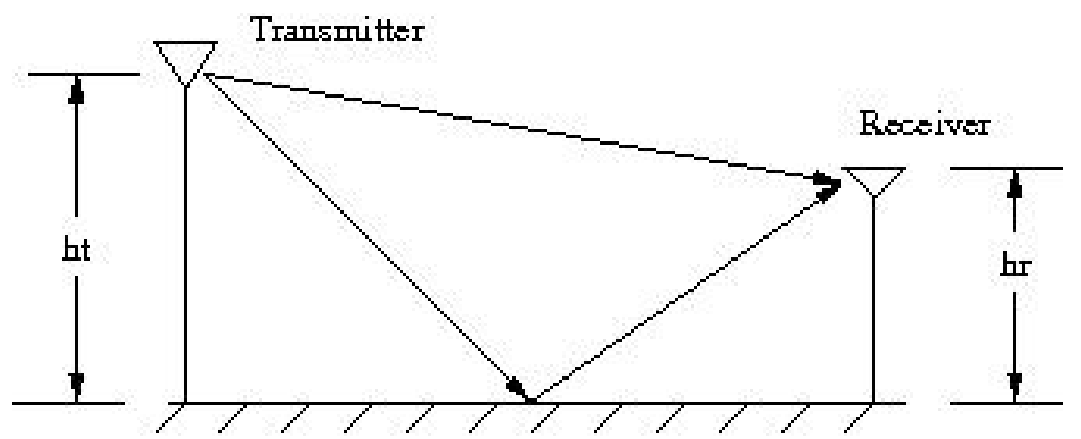

Figure 15: Two-ray propagation model.

The power loss relation in the far-field assuming the distance between two antennas is much greater than the product of the height of the antennas (ht,hr) is

$L(d B)=40 \log (d)+P_{\text {loss }}-(10 \log (G t)+10 \log (G r)+20 \log (h t)+20 \log (h r))$

Assuming unit directional antennas and $\mathrm{ht}=\mathrm{hr}=10 \mathrm{~m}$, we solve for the distance $(d$ ) that will give us a loss of $143 \mathrm{~dB}(30+113)$. In this case $d=11.7$ miles which is a significant reduction in range.

Typically there will not be a direct line of sight between the transmitter and receiver. Large scale fading refers to the modeling of the effects of prominent terrain contours (hills, buildings, etc.) between the transmitter and receiver. The path loss contribution to be factored into the link budget analysis is around $10 \mathrm{~dB}$ [Sklar 1997]. In this case the distance calculated from the equation above is around 6.5 miles. Small scale fading caused by time variation in the channel is small as the antennas are not mobile.

It should also be noted that the above calculations where with omni-directional antennas. Use of Yagi antenna's which have gains upto $13 \mathrm{dBi}$ will significantly increase the range. 


\subsubsection{Field Testing for Range}

Field test results were carried out to characterize the range of the Freewave FGR 900 $\mathrm{MHz}$ series radios with the help of INDOT personnel from Crawfordsville. One radio was fixed on the roof of the Civil Engineering building at Purdue University and the other radio was taken to several locations around West Lafayette. The goal was to characterize the range over which the radios can communicate by measuring their signal strength and data throughput.

In order to use Freewave's Diagnostic Program at the remote field location, the radio in Civil Engineering was configured as a point-to-multipoint slave and the remote radio was configured as a point-to-multipoint master. The two radios were set with the same network identification and frequency key.

At each test location the following information was recorded:

- The signal and noise strength measured by Freewave’s Diagnostic Program.

- Data throughput, bit error rate, and byte loss rate over five minute test using a program developed by a previous JTRP project.

The distance and elevation data in Table 4 were obtained using the U.S. Geological Survey (USGS) Topomap (http://nmviewogc.cr.usgs.gov/viewer.htm) since the readings given by the GPS receiver at the test sites were not sufficiently accurate. The equipment setup is shown in Figure 16. Test site locations are shown on an aerial photo (Figure 17) and a topological map (Figure 18). 


\begin{tabular}{|c|c|c|c|c|c|c|c|c|}
\hline $\begin{array}{l}\text { Site } \\
\text { ID }\end{array}$ & Location $^{1}$ & $\begin{array}{l}\text { Distance } \\
\text { from Civil } \\
\text { (mi) }\end{array}$ & $\begin{array}{l}\text { Elevation } \\
\text { (ft) }\end{array}$ & $\begin{array}{c}\text { Signal } \\
(\mathrm{J})^{2}\end{array}$ & $\begin{array}{l}\text { Noise } \\
\text { (J) }\end{array}$ & $\begin{array}{l}\text { Throughput } \\
\text { (bps) }\end{array}$ & $B E R^{3}$ & $\mathrm{BLR}^{4}$ \\
\hline 3 & $\begin{array}{c}\text { Turfgrass } \\
\text { Research } \\
\text { Center }\end{array}$ & 0.92 & 695 & 55 & 31 & 7570 & 0 & 0 \\
\hline 4 & Bowen Lab & 1.36 & 544 & 55 & 30 & 11100 & 0 & 0 \\
\hline 2 & Family Inn & 1.45 & 715 & $\star^{5}$ & & 264 & 0 & 0 \\
\hline 1 & Walmart & 2.26 & 689 & $\star * *^{6}$ & & & & \\
\hline 5 & $\begin{array}{l}\text { Reynolds and } \\
\text { Scott (Home } \\
\text { Hospital) }\end{array}$ & 2.57 & & 45 & 31 & 1202 & 0 & 0 \\
\hline 6 & $\begin{array}{c}\text { State } 26 \text { and } \\
52\end{array}$ & 3.10 & 679 & ** & & & & \\
\hline 7 & 231 and 25 & 3.17 & 620 & 55 & 30 & 3249 & 0 & 0 \\
\hline 8 & $\begin{array}{l}\text { Half mile from } \\
231 \text { and } 25\end{array}$ & 3.69 & 633 & 58 & 29 & 5949 & 0 & 0 \\
\hline 9 & $\begin{array}{l}1 / 3 \text { mile from } \\
231 \text { and } 350\end{array}$ & 4.30 & 573 & ** & & & & \\
\hline 10 & 231 and $400 S$ & 4.92 & 635 & ** & & & & \\
\hline
\end{tabular}

Table 4: Observed signal/noise strength and throughput.

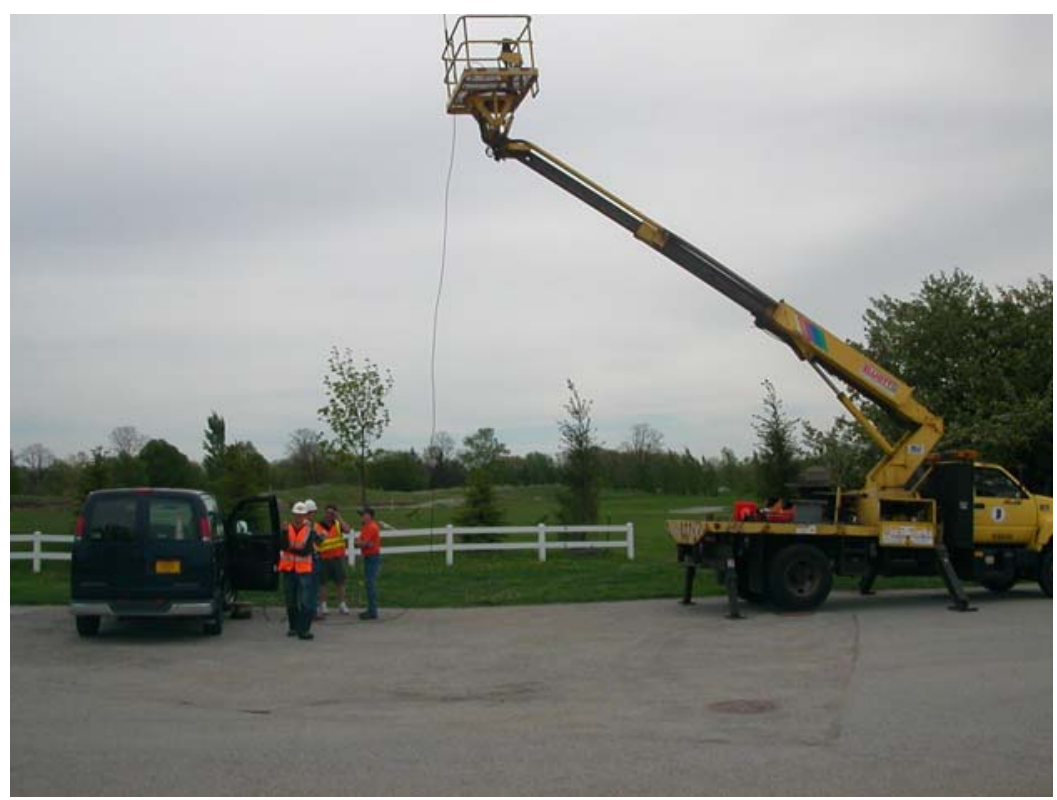

Figure 16: Remote radio equipment in van and antenna as hoisted by INDOT boom truck at test site number 3 .

\footnotetext{
${ }^{1}$ Locations are sorted by distance to the Civil Engineering building.

${ }^{2}$ Johnson's Factor (a parameter used by Freewave's diagnostic software. Note that RSSI $(\mathrm{dBm})=$ Johnson's Factor of signal or noise -150 .

${ }^{3}$ Bit error rate.

${ }^{4}$ Byte loss rate.

${ }^{5}$ Here signal strength was too low to be measured by the Freewave diagnostic program although the Purdue testing software could still be used to send data and measure throughput.

${ }^{6}$ Signal strength too low to measure or to send data with the Purdue testing software.
} 


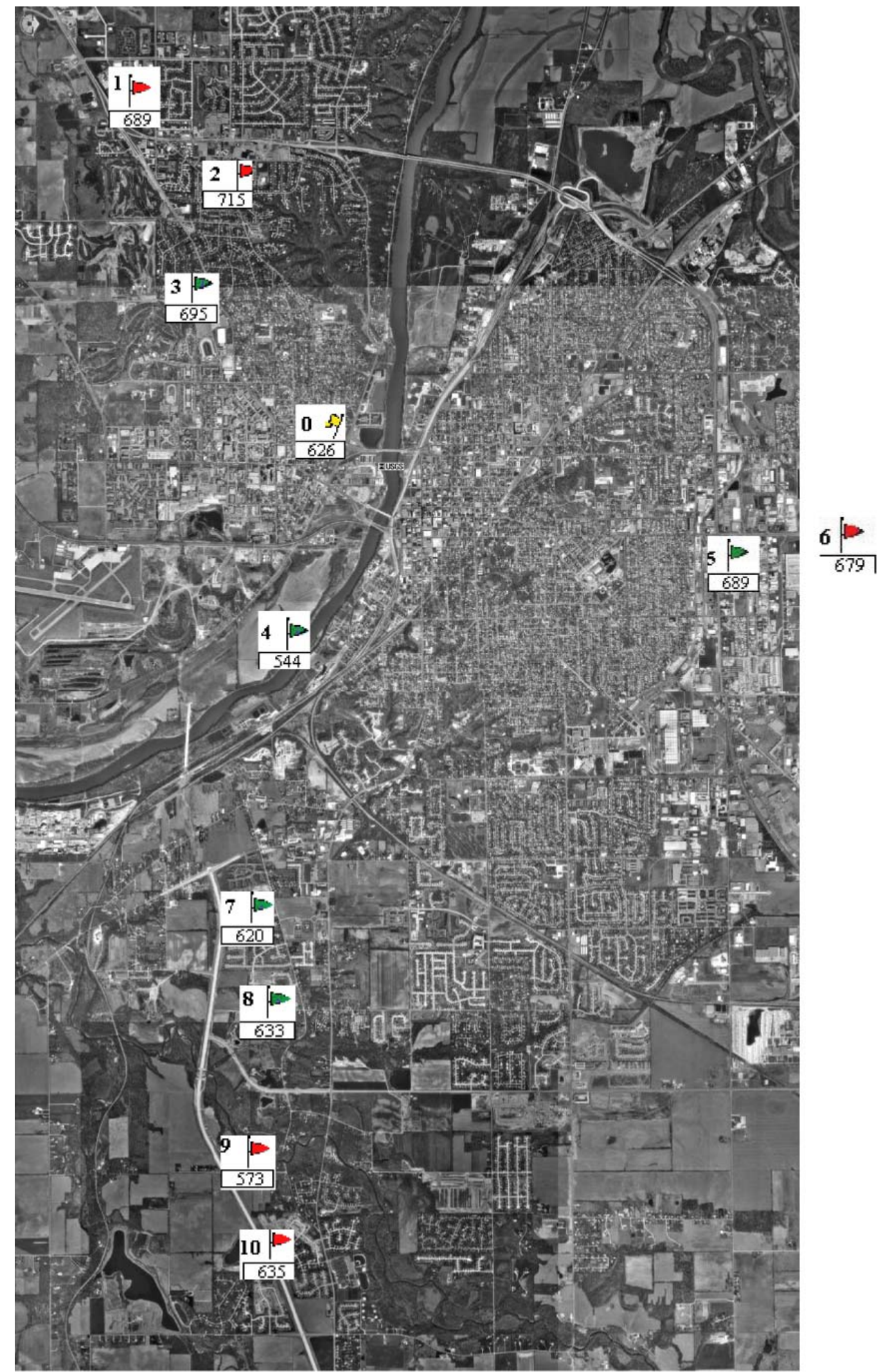

Figure 17: Aerial photo showing test sites. 


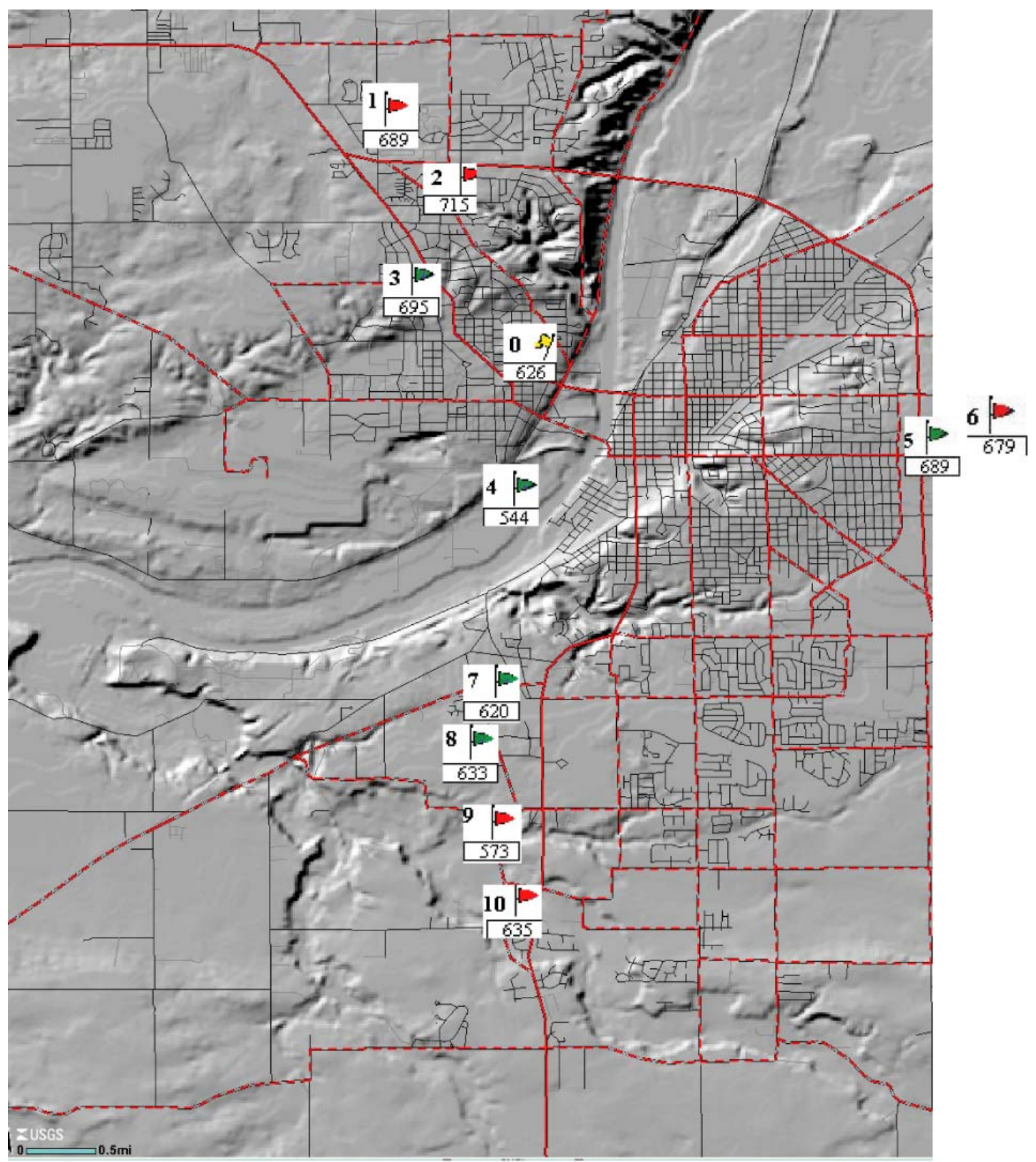

Figure 18: Topological map showing test sites. 


\section{Conclusions}

\subsection{Task A: Support for Field Tests of Satellite Communication Service Providers}

In the experiments conducted in Task A it can be seen that, by implementing the TCP splitting algorithm, certain applications can use the satellite bandwidth efficiently while others can not. SiteManager clients performed poorly in the high latency environment, in some cases up to 50 times slower in comparison to SiteManager running on a low latency terrestrial network with equivalent bandwidth. In general, the performance of SiteManager was relatively insensitive to the bandwidth provided by the satellite link. In fact, for most tasks, SiteManager performed better over a $50 \mathrm{kbps}$ dial up connection than over a 384 kbps satellite connection. An explanation has been given and the key to good performance lies in whether a program is written with the underlying network environment in mind. In the SM.07 procedure, which merely generates a blank form, if all 12 request/response pairs could be aggregated into 1, the response time would drop from 10 seconds to under 2 seconds. This "chatty" behavior can actually be found in other applications such as Microsoft Outlook where developers do not take the potentially high latency network into consideration when writing the program. In contrast, applications such as FTP work very will in high latency environments precisely because the application uses a small number of large packets instead of a large number of small packets.

In cases where applications are designed to have a lot of back and forth chatter between the client and the server, such as the AASHTO SiteManager or Microsoft Outlook, terminal emulation programs such as Citrix or VNC provide significant improvements in performance. In the case of applications such as Microsoft Outlook the chatter is relatively modest and the application can function without terminal emulation. However, in the case of AASHTO SiteManager it is virtually impossible to productively use the application in client-server mode over the high latency satellite connection. The only mechanism for providing acceptable SiteManager performance is to deploy it in an architecture where SiteManager is operated remotely via a terminal emulation. This architecture requires considerable more equipment, software, and technical support. Furthermore, the delay in seeing some keystrokes and cursor movements appear can be somewhat awkward for the user. However, given the extensive bursts of short messages between SiteManager clients and the server, the high latency constraints of a satellite network make a terminal emulation procedure the only viable method of deploying SiteManager via a commercial satellite IP service.

\subsection{Task B: Spread Spectrum Radio Testing}

Regarding the range of Freewave's radio technology, which is claimed to be 60 miles in line of sight conditions, we were unable to verify this claim either theoretically or by measurement. Common sense tells us that a 60 mile range in Indiana propagation 
environments with antenna heights corresponding to placement on signal arms is an order of magnitude too optimistic. In fact, our very rough calculations of link budget would suggest ranges more on the order of 10 miles and by measurement we were unable to attain ranges of more than about 3 miles. This stands to reason. Furthermore, with antenna heights of only 30 feet or so, line of sight conditions are very difficult to attain over more than a mile or two. Therefore, based only upon the range calculation, the idea of using a network of spread spectrum radios in a ad-hoc store and forward mode of operation to communicate long distances is impractical as nodes would have to be deployed much more densely than they are currently.

In addition, while the current generation of spread spectrum radios does offer vastly improved network flexibility it is still impractical to build a multihop network based upon this technology. Vendors do not support this mode of operation, which would therefore entail a significant effort on the part of any user to develop the software needed to control such a network. One would also expect delay through the network to grow significantly with the number of hops.

\section{Recommendations and Implementation Suggestions}

\subsection{Task A: Support for Field Tests of Satellite Communication Service Providers}

The analysis and test results obtained in Task A make it clear that a high bandwidth communications link with large delay is a very serious bottleneck to throughput when coupled with a client-server application that involves multiple client-server transactions to accomplish a single task. SiteManager is indeed such a "chatty" client-server application. Each transaction was seen to incur a delay corresponding to the roundtrip delay of the end-to-end communications link and when multiple transactions were required to complete a single task there was a large multiplier of this roundtrip delay as perceived by the user. SiteManager running on a client in the field was essentially useless when communicating over the satellite link. It should be noted that a satellite link is not the only example of a large delay link that could be proposed for INDOT communications although satellite provides the most extreme delay case that is likely to be seen. As seen in the backhaul delay from the satellite service provider to INDOT headquarters, a long Internet delay could easily reach round trip times of a quarter of a second, which, if multiplied by a "chatty" application, could still yield a significant reduction in throughput.

There are two possible solutions to the problem leading to our recommendations for implementation.

1. Improve the design of client-server software. By its very nature the work of a field engineer takes place from time to time in remote locations where communications must either be low bandwidth or high delay or both. In fact, the 
potential remoteness of the field engineer's worksite is one of the prime drivers of the idea of providing remote computing capability with a "fat" client communicating infrequently with a server at headquarters. Such software should not be designed to be "chatty" as it defeats the entire purpose. INDOT should work with other state DOTs to apply pressure to AASHTO to redesign the SiteManager application so that the advantages of having a "fat" client in the field can be attained with any available client-server communications link.

2. Use terminal emulation with client running in another location where it can be connected to the server by a high capacity, low delay link. Terminal emulation using a program such as Citrix was demonstrated to be a reasonable short term fix for the problems encountered. INDOT should pursue a Citrix or similar solution for the near term rollout of SiteManager. However, it should be noted that running the client software remotely from the field engineer has one serious disadvantage. With this method the field engineer must be able to communicate to headquarters from anywhere on the job site if he or she is to use the software away from the field office trailer. Some provision must be made for wireless Internet access along the length of the job site.

\subsection{Task B: Spread Spectrum Radio Testing}

Task B made use of a testbed network for experimentation with spread spectrum communications in the 900 and $2400 \mathrm{MHz}$ band, that was originally constructed with funding from a previous JTRP project. The current network consists of five fixed nodes and two portable nodes. Of the fixed nodes, three are located in the MSEE building, one is located in the Harold L. Michael Traffic Operations Laboratory, and one is located in the experimental traffic signal cabinet at the intersection of Stadium and Northwestern Avenues in West Lafayette. The testbed has been used to evaluate spread spectrum radio technologies from vendors Microwave Data Systems, GINA, EnCom, and Freewave.

In order to maximize the testbed's benefit to INDOT it is recommended that testbed operation be continued to allow further radio testing and experimentation with radio and traffic control and sensor integration. In this task, it is proposed to continue the operation of the testbed to accomplish the following:

1. Testing of improved vendor radios as they become available. In particular, the testbed could be used to evaluate new IP radios.

2. Experimentation with the setting of various radio network parameters (e.g., error control coding, data rate, retransmission protocols) and how they influence the performance of application specific software, such as traffic signal control.

3. Experimental verification of analytical models for network performance evaluation (e.g., throughput, etc.). 


\section{References}

[Allman 1999] M. Allman, “TCP Congestion Control,” Internet RFC 2581, 1999.

[Balakrishnan 2001] H. Balakrishnan and V. Padmanabhan, "How Network Asymmetry Affects TCP,” IEEE Communications Magazine, pages 60-67, April 2001.

[Bi 2002] Q. Bi and S. Vitebsky, "Performance Analysis of 3G-1X EVDO High Data Rate System," Proceedings of the Wireless Communications and Networking Conference (WCNC 2002), pages 389-395, March 2002.

[Braden 1994] R. Braden, "T/TCP - TCP Extensions for Transactions, Functional Specifications," Internet RFC 1644, 1994.

[Cohen 2003] M. Cohen, "Cost-Effective Microwave Solutions for Short and Medium Haul Networks," Technical paper available on Harris Microwave web site: http://www.microwave.harri.com/technologies/techpubs.html.

[EK 2002] Chapter 7: Communications Strategic Plan, Version 4.0, Indiana Department of Transportation Division of Systems Technology, October 28, 2002.

[EK 2003] Communications Planning and Coordination: Overview of Satellite Services, Pricing and Recommendations, Indiana Department of Transportation, April 30, 2003.

[Ghani 1999] N. Ghani and S. Dixit, “TCP/IP Enhancements for Satellite Networks,” IEEE Communications Magazine, pages 64-72, July 1999.

[Hackett 1981] C. M. Hackett, "An Efficient Algorithm for the Soft-Decision Decoding of the $(24,12)$ Extended Golay Code," IEEE Transactions on Communications, Vol. COM-29, No. 6, pages 909-911, June 1981.

[Henderson 1999] T. Henderson and R. Katz, "Transport Protocols for InternetCompatible Satellite Networks," IEEE Trans. Selected Areas in Communications, Vol. 17, pages 326-344, February 1999.

[Jacobson 1988] V. Jacobson, "Congestion Avoidance and Control," Proc. ACM SIGCOMM 1988 Conference, pages 314-329, 1988.

[Jacobson 1992] V. Jacobson, R. Braden and D. Borman, "TCP Extensions for High Performance,” Internet RFC 1323, 1992. 
[Krogmeier 2002] J. V. Krogmeier, "SPR-2395: Evaluation Procedures for Deploying Spread Spectrum Interconnect,” Report No. FHWA/IN/JTRP-2003/1, File No. 8-9-14, Joint Transportation Research Project - Purdue University, 2002.

[Krogmeier 1996] J. V. Krogmeier, K. C. Sinha, M. P. Fitz, S. Peeta, S. Y. Nof, "Borman Expressway ATMS Equipment Evaluation,” Report No. FHWA/IN/JHRP96/15, File No. 8-9-3, Joint Transportation Research Project - Purdue University, 1996.

[Lakshman 1997] T. Lakshman and U. Madhow, "The Performance of TCP/IP for Networks with High Bandwidth-Delay Products and Random Loss,” IEEE Trans. Networking, Vol. 5, pages 336-350, June 1997.

[Liu 2002] J. Liu and K. P. Worrall, "Theory and Practice of 3G Network Planning," Proceeding of the Third International Conference on 3G Mobile Communication Technologies, pages 74-80, May 2002.

[Mathis 1996] M. Mathis, J. Mahdavi, S. Floyd and A. Romanow, "TCP Selective Acknowledgment Options," Internet RFC 2018, 1996.

[Metz 1999] C. Metz, “TCP over Satellite,” IEEE Internet Computing, pages 76-80, January 1999.

[Mhatre 2002] V. Mhatre and C. Rosenberg, "Performance Improvement of TCP-based Applications in a Multi-access Satellite System," Proc. IEEE VTC, pages 1530-1534, September 2002.

[Mogul 1990] J. Mogul and S. Deering, "Path MTU Discovery," Internet RFC 1191, 1990.

[Muhonen 1998] J. Muhonen and R. Durst, "Performance of Transport Protocols Over Satellite Communication Links," IEEE Military Communications Conference (MILCOM), Vol. 1, pages 263-269, 1998.

[Partridge 1997] C. Partridge and T. Shepard, "TCP/IP Performance over Satellite Links,” IEEE Network, pages 44-49, September 1997.

[Philopoulos 2002] S. Philopoulos and K. Ferens, "Proxy-based Connection-splitting Architectures for Improving TCP Performance over Satellite Channels," Proc. IEEE Canadian Conf. on Electrical and Computer Engineering, pages 14301435, 2002.

[Pratt 2003] T. Pratt, C. Bostian, and J. Allnut, "Satellite Communications, Second Edition,” John Wiley and Sons, New Jersey, 2003.

[Samaraweera 1997] N. Samaraweera and G. Fairhurst, "Explicit Loss Indication and Accurate RTO Estimation for TCP Error Recovery using Satellite Links,” IEE Proc. Communication, Vol. 144, pages 44-53, February 1997. 
[Sklar 1997] B. Sklar, "Rayleigh Fading Channels in Mobile Digital Communication Systems Part 1: Characterization," IEEE Communications Magazine, pages 136-146, September 1997.

[Stevens 1996] W. Stevens, TCP/IP Illustrated, Addison-Wesley, 1996.

[Wang 2004] R. Wang, B. Gutha and R. Chandrasekaran, "Behavior of Vegas Congestion Control over Satellite Channels,” IEEE WCNC, pages 2027-2032, 2004. 